\title{
A Schrödinger approach to Newton-Cartan and Hořava-Lifshitz gravities
}

\author{
Hamid R. Afshar, ${ }^{a}$ Eric A. Bergshoeff, ${ }^{b}$ Aditya Mehra, ${ }^{c}$ Pulastya Parekh \\ and Blaise Rollier ${ }^{b}$ \\ ${ }^{a}$ School of Physics, Institute for Research in Fundamental Sciences (IPM), \\ P.O. Box 19395-5531, Tehran, Iran \\ ${ }^{b}$ Van Swinderen Institute for Particle Physics and Gravity, University of Groningen, \\ Nijenborgh 4, 9747 AG Groningen, The Netherlands \\ ${ }^{c}$ Indian Institute of Science Education and Research, \\ Dr Homi Bhabha Road, Pashan, Pune 411008, India \\ E-mail: afshar@ipm.ir, E.A.Bergshoeff@rug.nl, \\ aditya.mehra@students.iiserpune.ac.in, \\ pulastya.parekh@students.iiserpune.ac.in, B.R.Rollier@rug.nl
}

AbSTRACT: We define a 'non-relativistic conformal method', based on a Schrödinger algebra with critical exponent $z=2$, as the non-relativistic version of the relativistic conformal method. An important ingredient of this method is the occurrence of a complex compensating scalar field that transforms under both scale and central charge transformations. We apply this non-relativistic method to derive the curved space Newton-Cartan gravity equations of motion with twistless torsion. Moreover, we reproduce $z=2$ Hořava-Lifshitz gravity by classifying all possible Schrödinger invariant scalar field theories of a complex scalar up to second order in time derivatives.

KEywords: Classical Theories of Gravity, Gauge Symmetry

ARXiv EPRINT: 1512.06277 


\section{Contents}

1 Introduction 1

2 The relativistic conformal method 5

2.1 Einstein-Hilbert invariant 5

$\begin{array}{ll}2.2 \text { Higher derivative invariants } & 10\end{array}$

3 A non-relativistic conformal method $\quad 11$

3.1 Newton-Cartan variables 11

$\begin{array}{lll}3.2 & \text { Compensating scalar field } & 12\end{array}$

$\begin{array}{ll}3.3 & \text { Schrödinger gauge fields } \\ \end{array}$

4 Newton-Cartan gravity 16

$\begin{array}{ll}4.1 \text { Torsionless NCG } & 16\end{array}$

$\begin{array}{lll}4.2 & \text { Torsional NCG } & 18\end{array}$

5 Hořava-Lifshitz gravity $\quad 21$

5.1 Scalar coupled Schrödinger gravities 21

5.1.1 The potential terms 22

5.1.2 The kinetic terms 23

5.1.3 The curvature terms 26

5.2 Gauge fixing 28

5.3 Identification with Hořava-Lifshitz gravity 31

6 Conclusions $\quad 32$

A Schrödinger gravity $\quad 34$

B Scalar Schrödinger field theories $\quad 36$

B.1 Potential terms 38

B.2 Kinetic terms 39

\section{Introduction}

General Relativity as a locally Poincaré invariant theory of gravity has passed many experimental tests and is very successful in describing the gravitational interactions in our world. The locally supersymmetric extension of Poincaré gravity is called supergravity. Although supersymmetry has not yet been detected in any of the running experiments much effort has been devoted to construct supergravity invariants of all kinds of sort, with or without matter. An extremely useful technique to construct such invariants, is the (super)conformal 
method [1-4] where one makes use of compensating multiplets that transform under (super)conformal transformations - for an introduction see [5]. Gauge-fixing some of the components of the compensating multiplet, one gets rid of the redundant (super)conformal symmetries and obtains the desired (super-)Poincaré-invariant. One of the advantages of this method is that one can use the different (super)conformal multiplets as building blocks to construct the more complicated (super-)Poincaré invariants. In this work we apply a similar technique to obtain non-relativistic Galilean invariants.

In recent years, gravitational theories with non-relativistic symmetries have attracted renewed attention and have been widely studied from both theoretical and phenomenological points of views. Two famous examples of such non-relativistic theories are NewtonCartan (NC) gravity [6, 7] and Hořava-Lifshitz (HL) gravity $[8,9]$. Initially, NC gravity was developed as the generally covariant arbitrary frame reformulation of Newtonian gravity. Subsequent developments showed that NC gravity correctly describes the non-relativistic limit of General Relativity, see e.g. [10]. ${ }^{1}$ In a different context, in the search for a consistent theory of quantum gravity, HL gravity has been proposed as a possible UV completion of Einstein's theory. Hořava's theory is based on the so-called foliation preserving diffeomorphisms instead of the full diffeomorphisms of General Relativity. Following on Hořava's proposal, a vast literature explored the low-energy consistency and phenomenological viability of the theory, see $[11,12]$ for reviews. While HL gravity is rather unrelated to NC gravity as a gravitational theory, it has recently been shown [13] that HL gravity can be reformulated in the same geometric formulation as NC gravity, namely using NC geometry - see e.g. [14-16] for early works on the geometric structure of NC. More precisely, the so-called non-projectable and projectable versions of HL gravity correspond to an action made of a collection of higher-derivative invariants constructed out of the variables of NC geometry respectively with and without torsion [13].

Additional independent motivations for studying non-relativistic theories of gravity come from both the potential condensed matter applications and the developments in non-relativistic holography, initially studied in [17-24]. In particular, HL gravity is interesting from the holographic point of view since it provides an alternative way of realizing a non-relativistic holographic model where the bulk and the boundary have the same non-relativistic geometric structure $[25,26]$. This has recently been argued to be a very natural, and promising, approach in the context of certain non-relativistic field theories called Warped CFT's [27, 28]. In fact, many condensed matter systems are described by non-relativistic field theories and coupling these systems to non-relativistic backgrounds provides appropriate external sources conjugated to their conserved currents. In the context of NC geometry, the appropriate way of performing this coupling has been studied from the theoretical point of view [29-34] and typical condensed matter applications include, e.g., the description of the quantum Hall effect [35-38] and even aging in materials [39-46].

A crucial aspect of Newton-Cartan geometry, particularly relevant in the developments mentioned just above, is that the geometry can involve a non-vanishing torsion tensor. The Torsional Newton-Cartan (TNC) geometry was first observed in $[47,48]$ in the context of

\footnotetext{
${ }^{1}$ The underlying symmetries of NC gravity are described by the centrally extended Galilean algebra which is called the Bargmann algebra.
} 
Lifshitz holography ${ }^{2}$ where it arises as the boundary geometry of a specific model supporting $z=2$ Lifshitz solutions. Following [50] on the gauging of the Bargmann algebra, it was later shown that the TNC geometry can be obtained by gauging the Schrödinger algebra [51]. In this work, we will exclusively consider a specific type of torsion referred to as 'twistless torsion'.

The purpose of this paper is to develop a non-relativistic version of the conformal method mentioned at the beginning of this introduction and to illustrate it in two different contexts. We apply this method at the level of the equations of motion to find the curved space covariant NC gravity field equations with torsion and we apply it to re-derive $z=2$ HL gravity by reproducing the Galilean invariants constructed in [13]. We expect that this procedure will be very efficient if one considers more complicated models such as supersymmetric HL or NC gravity theories. In our formalism we use Schrödinger gravity, i.e. the gauge theory of the Schrödinger algebra $[52,53]$, as the analogue of conformal gravity in the relativistic case. We remind that the Schrödinger transformations are the maximal symmetries that leave invariant the action of a massive non-relativistic particle whereas the action of a massless non-relativistic particle is invariant under the symmetries of the so-called Galilean conformal algebra $[54,55]$. We will therefore reserve the name 'non-relativistic conformal gravity' for the gauge theory of the Galilean conformal algebra. ${ }^{3}$ For our present purposes, it is sufficient to make use of Schrödinger gravity.

The Schrödinger symmetries in $d$ spatial dimensions with critical exponent $z$, which we denote by $\operatorname{Sch}_{z}(d)$, contain in addition to the centrally extended Galilean symmetries, a dilatation generator $D$ which acts on space and time coordinates differently. To be precise, for general exponent $z$ the time and space coordinates transform under a dilatation with parameter $\lambda$ as follows:

$$
t \rightarrow \lambda^{z} t \quad \text { and } \quad \mathbf{x} \rightarrow \lambda \mathbf{x} .
$$

At $z=2$ an extra generator $K$, denoted as the generator of special conformal transformation, emerges. These two extra symmetries $D$ and $K$, together with the translations (with generators $P_{a}$ ), spatial rotations (with generators $J_{a b}$ ) and Galilean boosts (with generators $G_{a}$ ) are symmetries of the Schrödinger equation;

$$
\left(i \partial_{0}-\frac{1}{2 \mathrm{M}} \partial_{a}^{2}\right) \Psi(t, \mathbf{x})=0
$$

where $\mathrm{M}$ appears as the central term in the commutator of Galilean boosts and translations:

$$
\left[P_{a}, G_{b}\right]=\delta_{a b} \mathrm{M}
$$

The corresponding central charge transformation acts as a phase transformation on the complex scalar $\Psi$. In the context of the Schrödinger equation this symmetry corresponds to particle number conservation. The field theories invariant under Schrödinger symmetries have been studied in [56-58].

\footnotetext{
${ }^{2}$ See [49] for a recent review on Lifshitz holography.

${ }^{3}$ We thank J. Lukierski for a discussion on this point.
} 
The conformal method is based on a Stückelberg mechanism for a compensating scalar field involved in the conformal multiplet. It turns out that in the non-relativistic case we need to use a compensating complex scalar that transforms under dilatations and central charge transformations. This is different from the relativistic case where a real scalar is sufficient and the central charge transformations are absent. There is one more difference with the relativistic case: while in the relativistic case the special conformal transformations are described by a vector generator $K_{\mu}$, in the non-relativistic case we only have a scalar generator $K$. As a consequence of this, we cannot gauge away the space components $b_{a}$ of the dilatation gauge field in the non-relativistic case. These remnants of the dilatation gauge field are precisely the origin of torsion in NC gravity [51].

It is natural to apply the non-relativistic conformal method we develop in this work to NC gravity itself, in particular to obtain the extension with torsion. As far as we know, $\mathrm{NC}$ gravity cannot be derived from an action principle, at least not with the usual field content. We therefore apply the non-relativistic method at the level of the equations of motion and only consider equations of motion for the compensating scalar. It turns out that the non-relativistic conformal method in this case provides a very efficient way of constructing the equations of motion of NC gravity with torsion, a result that, as far as we know, has not occurred before in the literature.

We also apply the non-relativistic conformal method to Schrödinger invariant scalar field theories. By reproducing the Galilean invariants of [13], we show that Schrödinger field theories (SFT's) are naturally mapped to HL gravity. Since HL gravity contains higher derivatives we need to consider higher-derivative SFT's for the compensating complex scalar. For our purposes it is sufficient to classify all SFT's up to two derivatives in time and four derivatives in space. Following the same procedure as in the relativistic case, we will couple these SFT's to Schrödinger gravity and next gauge-fix the compensating complex scalar after which we obtain higher-derivative Galilean invariants.

This paper is organized as follows. In section 2 we review the relativistic conformal construction of Poincaré gravity. In section 3 we develop the non-relativistic conformal method that will be used in the remainder of the paper. We then use the non-relativistic conformal method to derive the Newton-Cartan equations of motion with and without torsion in section 4 . In section 5 we couple the complex compensating scalar to Schrödinger gravity, gauge fix the scalar and thereby construct all Galilean invariants with at most two time and four spatial derivatives that can be related to a SFT. This construction leads to a systematic derivation of Hořava-Lifshitz gravity at $z=2$. Finally, we present our conclusions in section 6. There are two appendices. In appendix A we give several details of the Schrödinger gravity theory, while in appendix B, we classify all scalar field theories that are invariant under rigid $z=2$ Schrödinger symmetries up to 2 derivatives in time and 4 derivatives in space.

Before starting we mention some notation and conventions. We work in $D=d+1$ spacetime dimensions where $d$ refers to the number of spatial dimensions. The small Latin alphabet letters $(a, b, c, \ldots)$ refer to the spatial local Galilean frame while the capitals $(\mathrm{A}, \mathrm{B}, \mathrm{C}, \ldots)$ cover the local Poincaré frame. The Greek indices $(\mu, \nu, \rho, \ldots)$ refer to the coordinate frame and labels all spacetime coordinates $(x \equiv t, \mathbf{x})$. 


\section{The relativistic conformal method}

Before discussing the non-relativistic case, it is instructive to first review the (bosonic) relativistic conformal construction. In the relativistic conformal construction the aim is to construct general Poincaré invariants by using the larger conformal symmetry algebra. The idea is that conformal field theories (CFT's) of a real scalar field correspond to a class of Poincaré invariants. The converse is not necessarily true, see below.

\subsection{Einstein-Hilbert invariant}

We explain first the relation between the Einstein-Hilbert invariant in $D>2$ dimensions and the CFT of a free real scalar with a standard 2-derivative kinetic term. To be explicit, we consider the Einstein-Hilbert Lagrangian in $D>2$ dimensions

$$
\mathrm{P}_{1}: \quad e^{-1} \mathcal{L}=\frac{1}{\kappa^{2}} \mathcal{R},
$$

where $e$ is the determinant of the Poincaré vielbein $e_{\mu}{ }^{\mathrm{A}}$ with $\mathrm{A}$ and $\mu$ referring to the local Poincaré and the coordinate frames respectively. The gravitational coupling constant $\kappa^{2}=16 \pi G$ has the length-dimension $D-2$. Clearly, the action corresponding to this Lagrangian is dimensionless and invariant under general coordinate transformations with parameter $\xi^{\mu}$ and under local Lorentz transformations with parameter $\Lambda^{\mathrm{AB}}=-\Lambda^{\mathrm{BA}}$ :

$$
\delta e_{\mu}{ }^{\mathrm{A}}=\xi^{\lambda} \partial_{\lambda} e_{\mu}{ }^{\mathrm{A}}+\left(\partial_{\mu} \xi^{\lambda}\right) e_{\lambda}^{\mathrm{A}}+\Lambda^{\mathrm{AB}} e_{\mu \mathrm{B}} .
$$

To relate the Poincaré invariant (2.1) to a real scalar field theory we first observe that the Einstein-Hilbert action is not invariant under local dilatations. To make it invariant under local dilatations we replace the Poincaré vielbein, which from now on we denote with $\left(e_{\mu}^{\mathrm{A}}\right)^{\mathrm{P}}$, by a compensating real scalar $\varphi$ times the conformal vielbein $\left(e_{\mu}{ }^{\mathrm{A}}\right)^{\mathrm{C}}$ :

$$
\left(e_{\mu}^{\mathrm{A}}\right)^{\mathrm{P}}=\kappa^{\frac{2}{D-2}} \varphi\left(e_{\mu}^{\mathrm{A}}\right)^{\mathrm{C}},
$$

where $\varphi$ has a lenght-dimension -1 . We inserted a factor with $\kappa$ in (2.3) so that the dilatation invariant action will be free of any dimensionful parameter under the field redefinition (2.3). The compensating scalar $\varphi$ and the conformal vielbein $\left(e_{\mu}{ }^{\mathrm{A}}\right)^{\mathrm{C}}$ transform under dilatations, with a local parameter $\Lambda_{\mathrm{D}}(x)$, such that the Poincaré vielbein $\left(e_{\mu}{ }^{\mathrm{A}}\right)^{\mathrm{P}}$ is invariant:

$$
\delta \varphi=-\Lambda_{\mathrm{D}} \varphi, \quad \delta\left(e_{\mu}{ }^{\mathrm{A}}\right)^{\mathrm{C}}=\Lambda_{\mathrm{D}}\left(e_{\mu}{ }^{\mathrm{A}}\right)^{\mathrm{C}} .
$$

We next substitute the decomposition (2.3) into the Poincaré invariant (2.1) and in this way end up with a real scalar coupled to conformal gravity. The corresponding action is invariant under general coordinate transformations, local Lorentz rotations and local dilatations. To obtain the conformal real scalar field theory, before coupling to conformal gravity, we impose the gauge-fixing condition ${ }^{4}$

$$
\left(e_{\mu}^{\mathrm{A}}\right)^{\mathrm{C}}=\delta_{\mu}{ }^{\mathrm{A}} .
$$

\footnotetext{
${ }^{4}$ Note that after gauge-fixing we do not distinguish between curved and flat indices anymore.
} 
This gauge-fixing condition restricts the local dilatation transformations to the rigid conformal transformations via the constraint equation

$$
\partial_{\mu} \xi^{\nu}+\Lambda_{\mu}^{\nu}+\Lambda_{\mathrm{D}} \delta_{\mu}^{\nu}=0
$$

This differential equation has the following solution

$$
\begin{aligned}
\xi^{\mu}(x) & =a^{\mu}-\lambda^{\mu \nu} x_{\nu}-\lambda_{\mathrm{D}} x^{\mu}+\lambda_{\mathrm{K}}^{\nu}\left(x^{\mu} x_{\nu}-\frac{1}{2} \delta_{\nu}^{\mu} x^{2}\right), \\
\Lambda^{\mu \nu}(x) & =\lambda^{\mu \nu}+2 \lambda_{\mathrm{K}}^{[\mu} x^{\nu]}, \\
\Lambda_{\mathrm{D}}(x) & =\lambda_{\mathrm{D}}-\lambda_{\mathrm{K}}^{\mu} x_{\mu},
\end{aligned}
$$

where $a^{\mu}, \lambda^{\mu \nu}, \lambda_{\mathrm{D}}$ and $\lambda_{\mathrm{K}}^{\mu}$ are the (constant) parameters of translations, Lorentz transformations, dilatations and special conformal transformations, respectively. The gauge-fixing condition (2.5) has the consequence that, when substituting expression (2.3) back into (2.1) one can ignore any derivative acting on the conformal vielbein. One thus ends up with a Lagrangian with the derivatives acting on the compensating scalar $\varphi$.

Finally, we make the redefinition (assuming $D>2$ )

$$
\varphi=\phi^{\frac{2}{D-2}}
$$

such that the Lagrangian (2.1) reduces to the following canonical form: ${ }^{5}$

$$
\mathrm{CFT}_{1}: \quad \mathcal{L}=4 \frac{D-1}{D-2} \phi \square \phi,
$$

where $\square=\eta^{\mu \nu} \partial_{\mu} \partial_{\nu}$ and $\eta^{\mu \nu}$ is the inverse flat Minkowski metric. The action corresponding to this Lagrangian in $D$ dimensions is explicitly invariant under the rigid conformal transformations

$$
\delta \phi=\xi^{\mu} \partial_{\mu} \phi+w \Lambda_{\mathrm{D}} \phi
$$

with $\xi^{\mu}$ and $\Lambda_{\mathrm{D}}$ given in (2.7) and, due to the redefinition (2.8), with weight $w$ given by

$$
w=-\frac{1}{2}(D-2) .
$$

We thus have shown how the Poincaré invariant $\mathrm{P}_{1}$ given in (2.1) can be related to the conformal field theory $\mathrm{CFT}_{1}$ of a free real scalar defined in (2.9).

The relation also works the other way around. Starting from the $\mathrm{CFT}_{1}$ given in (2.9) one can derive the Einstein-Hilbert Lagrangian (2.1) as follows. The first step is to make the $\mathrm{CFT}_{1}$ of (2.9) invariant under local conformal transformations, i.e. couple it to conformal gravity. In order to do this, it is convenient to first introduce all the gauge fields of conformal gravity, not only the conformal vielbein. By applying a standard gauging procedure to

\footnotetext{
${ }^{5}$ As a general feature a positive kinetic term for the compensating scalar corresponds to a negative kinetic term for gravity and vice-versa [5].
} 
the relativistic conformal algebra, see e.g. [5] and references therein, ${ }^{6}$ one arrives at the following gauge fields and transformation rules - we omit the superscript $\mathrm{C}$ from now on;

$$
\begin{aligned}
\delta e_{\mu}{ }^{\mathrm{A}} & =\Lambda^{\mathrm{AB}} e_{\mu}{ }^{\mathrm{B}}+\Lambda_{\mathrm{D}} e_{\mu}{ }^{\mathrm{A}}, \\
\delta \omega_{\mu}{ }^{\mathrm{AB}} & =D_{\mu} \Lambda^{\mathrm{AB}}+4 \Lambda_{\mathrm{K}}^{[\mathrm{A}} e_{\mu}{ }^{\mathrm{B}]}, \\
\delta b_{\mu} & =\partial_{\mu} \Lambda_{\mathrm{D}}-2 \Lambda_{\mathrm{K}}^{\mathrm{A}} e_{\mu \mathrm{A}}, \\
\delta f_{\mu}{ }^{\mathrm{A}} & =D_{\mu} \Lambda_{\mathrm{K}}^{\mathrm{A}}+\Lambda^{\mathrm{AB}} f_{\mu \mathrm{B}}+b_{\mu} \Lambda_{\mathrm{K}}^{\mathrm{A}}-\Lambda_{\mathrm{D}} f_{\mu}{ }^{\mathrm{A}},
\end{aligned}
$$

where $D_{\mu}$ denotes the Lorentz-covariant derivative and $\Lambda_{\mathrm{K}}^{\mathrm{A}}$ is the parameter of a special conformal transformation. All gauge fields transform as covariant vectors under general coordinate transformations.

The special thing about the three new gauge fields that we have introduced is that two of them, $\omega_{\mu}{ }^{\mathrm{AB}}$ and $f_{\mu}^{\mathrm{A}}$, are dependent, i.e. $\omega_{\mu}^{\mathrm{AB}}=\omega_{\mu}{ }^{\mathrm{AB}}(e, b)$ and $f_{\mu}{ }^{\mathrm{A}}=f_{\mu}^{\mathrm{A}}(e, b)$, whereas the third gauge field $b_{\mu}$ transform as a shift under special conformal transformations, see eq. (2.12c). The fact that the gauge field $b_{\mu}$ is the only field that transforms with a shift under the special conformal transformations ${ }^{7}$ means that, writing out all covariant derivatives, one finds that the dilatation gauge field always drops out in any conformal invariant action in $D>2$.

Despite the fact that $b_{\mu}$ does not occur in the final answer, it is useful to keep this gauge field at a first stage to couple the real scalar conformal field theory (2.9) to conformal gravity and construct covariant derivatives in a systematic way. This goes as follows. Since the scalar field $\phi$ only transforms under general coordinate transformations and dilatations it's conformal covariant derivative is defined as follows:

$$
D_{\mathrm{A}} \phi=e_{\mathrm{A}}{ }^{\mu}\left(\partial_{\mu}-w b_{\mu}\right) \phi .
$$

Due to the presence of the dilatation gauge field $b_{\mu}$ this conformal covariant derivative transforms under special conformal transformations and, therefore, if we take the conformal covariant derivative of the expression (2.13) it will involve the gauge field of special conformal transformations. The exact expression for the conformal d'Alembertian reads:

$$
\begin{aligned}
\square^{\mathrm{C}} \phi & \equiv \eta^{\mathrm{AB}} D_{\mathrm{A}} D_{\mathrm{B}} \phi \\
& =e^{\mathrm{A} \mu}\left(\partial_{\mu} D_{\mathrm{A}} \phi-(w-1) b_{\mu} D_{\mathrm{A}} \phi-\omega_{\mu \mathrm{A}}{ }^{\mathrm{B}}(e, b) D_{\mathrm{B}} \phi-2 w f_{\mu \mathrm{A}}(e, b) \phi\right) .
\end{aligned}
$$

The Lagrangian describing the coupling of $\phi$ to conformal gravity is then given by

$$
e^{-1} \mathcal{L}=4 \frac{D-1}{D-2} \phi \square^{\mathrm{C}} \phi .
$$

The theory described by this Lagrangian is invariant under the local conformal transformations (2.10), where $\xi^{\mu}$ and $\Lambda_{\mathrm{D}}$ are now taken to be arbitrary functions of the spacetime

\footnotetext{
${ }^{6}$ Note that our sign conventions are different from [5].

${ }^{7}$ The gauge fields $\omega_{\mu}{ }^{\mathrm{AB}}(e, b)$ and $f_{\mu}{ }^{\mathrm{A}}(e, b)$ transform only under special conformal transformations via their dependence on $b_{\mu}$.
} 
coordinates, and (2.12) provided that the scalar weight $w$ is given by (2.11) - see [5] for more details.

To obtain the Einstein-Hilbert action we now fix the dilatations by imposing the gaugefixing condition

$$
\phi=\frac{1}{\kappa}
$$

Note that this gauge-fixing condition does not require any compensating transformations. As we already mentioned, the $b_{\mu}$ gauge field will automatically drop out from (2.15) as a consequence of the special conformal invariance. Therefore, from the expression (2.14) it is clear that the $b_{\mu}$ independent part of $e_{\mathrm{A}}{ }^{\mu} f_{\mu}{ }^{\mathrm{A}}$ is the only relevant term that will ultimately survive the gauge-fixing condition (2.16). Using the explicit expressions for $\omega_{\mu}{ }^{\mathrm{AB}}$ and $f_{\mu}{ }^{\mathrm{A}}$,

$$
\begin{aligned}
\omega_{\mu}{ }^{\mathrm{AB}}(e, b) & =\Omega_{\mu}{ }^{\mathrm{AB}}(e)+2 e_{\mu}{ }^{[\mathrm{A}} e^{\mathrm{B}] \nu} b_{\nu}, \\
f_{\mu}{ }^{\mathrm{A}}(e, b) & =\frac{1}{2(D-2)}\left(R_{\mu}{ }^{\mathrm{A}}(\omega)-\frac{1}{2(D-1)} e_{\mu}{ }^{\mathrm{A}} R(\omega)\right),
\end{aligned}
$$

with

$$
\begin{aligned}
\Omega_{\mu}{ }^{\mathrm{AB}}(e) & =-2 e^{\nu[\mathrm{A}} \partial_{[\mu} e_{\nu]}^{\mathrm{B}]}+e_{\mu \mathrm{C}} e^{\nu \mathrm{A}} e^{\rho \mathrm{B}} \partial_{[\nu} e_{\rho]}^{\mathrm{C}}, \\
R_{\mu}{ }^{\mathrm{A}}(\omega) & =2 e_{\mathrm{B}}{ }^{\nu}\left(\partial_{[\mu} \omega_{\nu]}{ }^{\mathrm{AB}}-\eta_{\mathrm{CD}} \omega_{[\mu}^{{ }^{\mathrm{AC}}} \omega_{\nu]}{ }^{\mathrm{DB}}\right)
\end{aligned}
$$

and $R(\omega)=e_{\mathrm{A}}{ }^{\mu} R_{\mu}{ }^{\mathrm{A}}(\omega)$, one finds that after gauge-fixing the matter coupled conformal gravity Lagrangian (2.15) precisely reduces to the Einstein-Hilbert Lagrangian (2.1) where the Poincaŕe Ricci scalar is

$$
\mathcal{R}=2 e_{\mathrm{A}}^{\mu} e_{\mathrm{B}}^{\nu}\left(\partial_{[\mu} \Omega_{\nu]}^{\mathrm{AB}}-\eta_{\mathrm{CD}} \Omega_{[\mu}^{\mathrm{AC}} \Omega_{\nu]}^{\mathrm{DB}}\right)
$$

This concludes our discussion of how the Einstein-Hilbert invariant is related to the CFT of a free real scalar with a 2-derivative kinetic term. A few remarks are in order. First of all, we note that in the relativistic case the number of derivatives in the Poincaréinvariant is the same as the number of derivatives in the corresponding CFT. As we will see later on, this will no longer be true in the non-relativistic case. Secondly, the way we couple the scalar to conformal gravity is basically by replacing derivatives by conformalcovariant derivatives. This only works nicely if we perform the covariantization on the Lagrangian (2.9). For instance, had we used as the CFT Lagrangian $\mathcal{L}^{\prime} \sim \partial_{\mu} \phi \partial^{\mu} \phi$ instead of $\mathcal{L} \sim \phi \square \phi$ we would not have succeeded to couple $\mathcal{L}^{\prime}$ to conformal gravity. The reason is that, although $\mathcal{L}$ and $\mathcal{L}^{\prime}$ only differ by a total derivative, and therefore are equivalent as CFT's, this total derivative ceases to be a total derivative after replacing derivatives by covariant derivatives. Hence, to distinguish between these two cases it is necessary to formulate the invariance directly at the level of the Lagrangian.

Under the variation $(2.10)$ neither $\mathcal{L}$ nor $\mathcal{L}^{\prime}$ are exactly invariant. Instead, they are both invariant up to a total derivative. Because ultimately we are interested in coupling these Lagrangians to conformal gravity, and anticipating on their future invariance under 
arbitrary coordinate transformations, we restrict the allowed total derivative and define an invariant Lagrangian as a Lagrangian whose variation is

$$
\delta \mathcal{L}=\partial_{\mu}\left(\xi^{\mu} \mathcal{L}\right)
$$

The rule is then that we can only couple those Lagrangians to conformal gravity that are invariant by themselves in the sense of (2.22). Note that in the case discussed above $\delta \mathcal{L}^{\prime} \neq \partial_{\mu}\left(\xi^{\mu} \mathcal{L}^{\prime}\right)$. In the remaining, when we talk about an invariant Lagrangian we will always mean invariance up to the total derivative (2.22).

As a last remark, let us stress that the relation between Poincaré invariants and scalar CFT's is not one-to-one. Although to each independent CFT we can associate a unique (linearly independent) Poincaré invariant, there exist Poincaré invariants that do not have a corresponding scalar CFT. Furthermore, not every CFT corresponding to a Poincaréinvariant has a kinetic term. The latter situation is what we denote as 'potential terms' and the former as 'curvature terms'. Accordingly, in the remainder of this paper we will distinguish between three different categories of CFT's/spacetime invariants:

1. Potential terms. Not every spacetime invariant corresponds to a CFT with a kinetic term. For instance, a cosmological constant term $\Lambda$ corresponds to a CFT without a kinetic term. Explicitly, we have

$$
\mathrm{CFT}_{0}: \quad \mathcal{L}=\Lambda \phi^{2}, \quad w=-\frac{D}{2} \quad \Leftrightarrow \quad \mathrm{P}_{0}: e^{-1} \mathcal{L}=\Lambda
$$

where, for clarity, we set $\kappa=1$ from now on. Note that in this case the field redefinition (2.8) is modified, as can be seen from the value of dilatation weight.

2. Kinetic terms. This category includes all CFT's with time derivatives. An example is the CFT (2.9) corresponding to the Einstein-Hilbert invariant. One can also consider higher-derivative CFT's corresponding to (linear combinations of) the invariants $R^{\mu \nu} R_{\mu \nu}$ and $R^{2}$ as will be discussed below.

3. Curvature terms. There are spacetime invariants that are not encoded by any corresponding CFT, examples include the Riemann curvature squared and relatedly the Weyl tensor squared. For instance, starting from the Weyl tensor squared in $D$ dimensions and making the redefinition (2.3) one ends up with the following term:

$$
e^{-1} \mathcal{L} \sim \phi^{2 \frac{D-4}{D-2}}\left(C_{\mu \nu}{ }^{\mathrm{AB}}\right)^{2}
$$

Clearly, upon gauge-fixing $e_{\mu}{ }^{\mathrm{A}}=\delta_{\mu}{ }^{\mathrm{A}}$, this term vanishes and therefore does not lead to any non-trivial CFT. We call this type of Poincaré invariants 'curvature terms' because they are related to the conformal invariants that can be built out of the conformal curvature. 


\subsection{Higher derivative invariants}

The procedure outlined above can easily be extended to include higher-derivative Poincaré invariants such as $R^{2}$ and $R^{\mu \nu} R_{\mu \nu} .{ }^{8}$ In that case one ends up with a higher-derivative scalar CFT. For completeness, and in order to better illustrate some of the points just mentioned, we briefly discuss the Poincaré invariants corresponding to the CFTs with four derivatives. Assuming $D>4$, we consider in this case a compensating scalar $\phi$ of dilatation weight

$$
w=-\frac{1}{2}(D-4) .
$$

Given this weight, it follows that the higher derivative field theory built out of the operator box squared, $\mathcal{L} \sim \phi \square^{2} \phi$, is a CFT. We call it $\mathrm{CFT}_{2}$ below. Using the general procedure outlined above, it can be seen to correspond to the following combination of the Ricci tensor squared and the Ricci scalar squared:

$$
\mathrm{CFT}_{2}: \quad \mathcal{L}=-\frac{(D-2)^{2}}{D-4} \phi \square^{2} \phi \quad \Leftrightarrow \quad \mathrm{P}_{2}: \quad e^{-1} \mathcal{L}=R^{\mu \nu} R_{\mu \nu}-\frac{D^{3}-4(D-2)^{2}}{16(D-1)^{2}} R^{2} .
$$

The fact that both the Ricci tensor squared and the Ricci scalar squared are separately invariant indicates that there is another independent CFT at that order. Starting from the Poincaré invariants $R^{\mu \nu} R_{\mu \nu}$ and $R^{2}$ separately the corresponding CFTs could be obtained by introducing the compensating scalar via a similar decomposition as we did in (2.3) for the Einstein-Hilbert term. Here, in analogy to what we will do in the non-relativistic case, we discuss a different way to obtain this result by starting from the CFT point of view.

First, note that up to a partial differentiation, the CFT considered in (2.26) is equivalent to the Lagrangian $\mathcal{L}^{\prime} \sim(\square \phi)^{2}$. The variation of Lagrangian $\mathcal{L}^{\prime}$ is proportional to $\square \phi$ which is the conformal scalar theory extensively discussed in section 2.1, and obviously cannot be coupled to conformal gravity given the weight $(2.25)$. However, $\mathcal{L}^{\prime}$ can be made invariant by adding compensating terms. ${ }^{9}$ From this we learn that there exists another CFT, denoted below as $\mathrm{CFT}_{3}$, which can be coupled to conformal gravity and, with appropriate normalisation, correspond to the Poincaré invariant $R^{2}$ after gauge-fixing:

$$
\mathrm{CFT}_{3}: \quad \mathcal{L}=16 \frac{(D-1)^{2}}{(D-4)^{2}}\left(\square \phi+\frac{2}{(D-4)} \frac{\partial_{\mu} \phi \partial^{\mu} \phi}{\phi}\right)^{2} \quad \Leftrightarrow \quad \mathrm{P}_{3}: \quad e^{-1} \mathcal{L}=R^{2} .
$$

The Lagrangians (2.26) and (2.27) describe two independent scalar CFTs. They are the only two invariant Lagrangians with four derivatives acting on the scalar $\phi$ of fixed dilatation weight (2.25). It is possible to obtain them starting from the most general linear combination of all possible terms that can be written down with four derivatives that have

\footnotetext{
${ }^{8}$ The same does not apply to the Riemann curvature squared term since that corresponds, using the terminology introduced above, to a curvature term.

${ }^{9}$ We thank Mehmet Özkan for useful comments on this construction.
} 
the correct scaling behaviour and by requiring that under the rigid conformal transformations the Lagrangian transforms according to (2.22). We thus find that, up to the curvature terms discussed above, a classification of all inequivalent CFTs correspond to all possible Poincaré invariants.

This concludes our review of the relativistic conformal construction. In the next section we will discuss what happens in the non-relativistic case.

\section{A non-relativistic conformal method}

In section 2 we reviewed how the Einstein-Hilbert action arises from a CFT of a free scalar with a two-derivative kinetic term. Like the Poincaré group which can be extended to the conformal group, the centrally extended Galilei group can be extended to the Schrödinger group. This prepares the base for our non-relativistic conformal construction both of Lagrangians that are invariant under local Galilean symmetries as well as of field equations that are covariant under these symmetries. The latter leads to the construction of the curved space Newton-Cartan Gravity (NCG) equations of motion with torsion discussed in section 4 while the former leads to the construction of Galilean invariants in section 5 and their physical realization as Hořava-Lifshitz Gravity (HLG) discussed in section 5.3.

\subsection{Newton-Cartan variables}

In general, the gauging of the Galilean algebra $[13,50]$ leads to a set of independent gauge fields which are given by a time-like vielbein $\tau_{\mu}$ and a spatial vielbein $e_{\mu}{ }^{a}$ - with $a$ referring to the spatial local Galilean frame, $a=1, \cdots, d$ - which obey the following transformation rules

$$
\begin{aligned}
\delta \tau_{\mu} & =0, \\
\delta e_{\mu}{ }^{a} & =\Lambda^{a b} e_{\mu}{ }^{b}+\Lambda^{a} \tau_{\mu},
\end{aligned}
$$

where $\Lambda^{a b}$ and $\Lambda^{a}$ are the parameters of a (local) spatial rotation and a Galilean boost, respectively. ${ }^{10}$ Furthermore, both gauge fields transform as covariant vectors under general coordinate transformations with parameter $\xi^{\mu}$. The inverses $\tau^{\mu}$ and $e^{\mu}{ }_{a}$ are defined by

$$
\begin{aligned}
\tau^{\mu} \tau_{\mu} & =1, & \tau^{\mu} e_{\mu}{ }^{a} & =0, \\
\tau_{\mu} e^{\mu}{ }_{a} & =0, & e_{\mu}{ }^{a} e^{\mu}{ }_{b} & =\delta_{b}^{a} .
\end{aligned}
$$

They obey the following transformation rules:

$$
\begin{aligned}
\delta \tau^{\mu} & =-\Lambda^{a} e^{\mu}{ }_{a}, \\
\delta e^{\mu}{ }_{a} & =\Lambda_{a b} e^{\mu}{ }_{b},
\end{aligned}
$$

and can be used to form the temporal and spatial projections of a given tensor $T_{\mu}$;

$$
T_{0} \equiv \tau^{\mu} T_{\mu} \quad \text { and } \quad T_{a} \equiv e_{a}^{\mu} T_{\mu} \quad \text { such that } \quad T_{\mu}=T_{0} \tau_{\mu}+T_{a} e_{\mu}^{a} .
$$

We will extensively use this notation below.

\footnotetext{
${ }^{10}$ Recent approaches for localizing the Galilean symmetry of a field theoretic model have been developed in $[59,60]$.
} 
The geometric realization of these variables is given in terms of the Newton-Cartan geometry which describes a non-relativistic spacetime. A difference with the relativistic case is that one cannot define a metric for the full spacetime. Requiring Galilean invariance one can only define a metric $\tau_{\mu \nu}$ in the time direction and a metric $h^{\mu \nu}$ in the spatial directions separately:

$$
\tau_{\mu \nu}=\tau_{\mu} \tau_{\nu}, \quad h^{\mu \nu}=e^{\mu}{ }_{a} e^{\nu}{ }_{b} \delta^{a b} .
$$

To define a metric with upper indices in the time direction and a metric with lower indices in the spatial directions that is invariant under Galilean boosts one needs a vector field that transforms under Galilean boosts with a shift [13]:

$$
\delta M_{\mu}=\Lambda_{a} e_{\mu}{ }^{a} .
$$

This vector field should be considered together with $\tau_{\mu}$ and $e_{\mu}{ }^{a}$ to define the full NewtonCartan geometry. In fact, using this vector field we can simply define the following boost invariant hatted variables:

$$
\begin{gathered}
\hat{\tau}^{\mu}=\tau^{\mu}+e^{\mu a} M_{a}, \\
\hat{e}_{\mu}{ }^{a}=e_{\mu}{ }^{a}-\tau_{\mu} M^{a} .
\end{gathered}
$$

Note that this basis preserves all the orthonormality conditions of (3.2).

The vector field $M_{\mu}$ can be promoted to a gauge field $m_{\mu}$ by a Stückelberg mechanism as we will see later on. The gauge field $m_{\mu}$ is associated to the central charge transformation in the centrally extended Galilean algebra, i.e. the Bargmann algebra [50]. There are several reasons to work with the Bargmann algebra rather than Galilean algebra:

1. The Lagrangian of a non-relativistic particle is only invariant under Galilean boosts up to a total derivative. This leads to a centrally extended Galilean algebra.

2. A vector field is needed to solve for the connection fields of spatial rotations and Galilean boosts in terms of the other gauge fields [50].

3. The vector field is needed to define a metric with upper indices in the time direction and a metric with lower indices in the spatial directions as stated above in eq. (3.7).

In this section we do not work with the Bargmann algebra but with the Schödinger algebra which is a minimal extension of it that serves our purposes. Therefore, the gauge field $m_{\mu}$ we will be using has a Schrödinger origin. More information about Schrödinger gravity and the gauging of the Schrödinger algebra can be found in appendix A.

\subsection{Compensating scalar field}

In order to relate a Galilean invariant to a Schrödinger invariant field theory we need to introduce compensating fields. Since we add two extra symmetries, dilatations and central charge transformations, we introduce two real scalars $\varphi$ and $\chi$ or, equivalently, a complex scalar $\Psi$ :

$$
\Psi=\varphi e^{i \chi}
$$


Any Galilean invariant can then be made invariant under local dilatations and central charge transformations by replacing the Galilean vielbein fields, which from now on we give a superscript G, by Schrödinger vielbein fields, which we indicate with a superscript Sch, and by replacing the vector field $M_{\mu}$ by the Schrödinger gauge field $m_{\mu}$ as follows:

$$
\begin{aligned}
\left(\tau_{\mu}\right)^{\mathrm{G}} & =\varphi^{-\frac{2}{w}}\left(\tau_{\mu}\right)^{\mathrm{Sch}}, \quad\left(e_{\mu}{ }^{a}\right)^{\mathrm{G}}=\varphi^{-\frac{1}{w}}\left(e_{\mu}{ }^{a}\right)^{\mathrm{Sch}}, \\
M_{\mu} & =m_{\mu}-\frac{1}{\mathrm{M}} \partial_{\mu} \chi,
\end{aligned}
$$

where $\mathrm{M}$ is an arbitrary parameter which is inserted to adjust the mass dimension and the dilatation weight $w$ is fixed up to a field redefinition ambiguity which is removed once we fix the invariant theory for $\Psi$, see section 5 . Here we use an arbitrary weight for the scalar field to avoid further field redefinition and to harmonize the notation with the next sections. It is important to note that we have used different scalings for $\tau_{\mu}$ and $e_{\mu}{ }^{a}$ in eqs. (3.9a) since we want to consider the case of Schrödinger gravity at $z=2$, see appendix A.

The two compensating scalars $\varphi$ and $\chi$, the Schrödinger vielbein gauge fields $\left(\tau_{\mu}\right)^{\text {Sch }}$, $\left(e_{\mu}{ }^{a}\right)^{\text {Sch }}$ and the Schrödinger central charge gauge field $m_{\mu}$ transform under spatial rotations and Galilean boosts like the fields $\left(\tau_{\mu}\right)^{\mathrm{G}},\left(e_{\mu}{ }^{a}\right)^{\mathrm{G}}$ and $M_{\mu}$. Furthermore, they transform under dilatations, with parameter $\Lambda_{\mathrm{D}}$, and central charge transformations, with parameter $\sigma$, such that the left-hand-side of (3.9) is invariant under these transformations:

$$
\begin{aligned}
\delta \Psi & =\left(w \Lambda_{\mathrm{D}}+i \mathrm{M} \sigma\right) \Psi, \\
\delta\left(\tau_{\mu}\right)^{\mathrm{Sch}} & =2 \Lambda_{\mathrm{D}}\left(\tau_{\mu}\right)^{\mathrm{Sch}}, \quad \delta\left(e_{\mu}{ }^{a}\right)^{\mathrm{Sch}}=\Lambda_{\mathrm{D}}\left(e_{\mu}{ }^{a}\right)^{\mathrm{Sch}}, \\
\delta m_{\mu} & =\partial_{\mu} \sigma .
\end{aligned}
$$

After substituting the decompositions (3.9) back into the Galilean invariant we end up with an action that describes the coupling of a complex scalar $\Psi$ to Schrödinger gravity. To obtain the complex scalar field theory before coupling to Schrödinger gravity we impose the following gauge fixing conditions:

$$
\left(\tau_{\mu}\right)^{\mathrm{Sch}}=\delta_{\mu 0}, \quad\left(e_{\mu}{ }^{a}\right)^{\mathrm{Sch}}=\delta_{\mu}{ }^{a}, \quad m_{\mu}=0,
$$

after which we do not distinguish between flat and curved indices anymore. These gaugefixing conditions imply the following constraint equations for the symmetry parameters:

$$
\begin{aligned}
\partial_{\mu} \xi^{0}+2 \Lambda_{\mathrm{D}} \delta_{\mu 0} & =0 \\
\partial_{\mu} \xi^{a}+\Lambda^{a b} \delta_{\mu}{ }^{b}+\Lambda^{a} \delta_{\mu 0}+\Lambda_{\mathrm{D}} \delta_{\mu}{ }^{a} & =0 \\
\partial_{\mu} \sigma+\Lambda_{a} \delta_{\mu}{ }^{a} & =0
\end{aligned}
$$


which can be solved in terms of the following rigid Schrödinger transformations:

$$
\begin{aligned}
\xi^{0}(t) & =a^{0}-2 \lambda_{\mathrm{D}} t+\lambda_{\mathrm{K}} t^{2}, \\
\xi^{c}(t, \mathbf{x}) & =a^{c}-\lambda^{c b} x^{b}-\lambda^{c} t-\lambda_{\mathrm{D}} x^{c}+\lambda_{\mathrm{K}} t x^{c}, \\
\Lambda^{a b} & =\lambda^{a b} \\
\Lambda^{a}(\mathbf{x}) & =\lambda^{a}-\lambda_{\mathrm{K}} x^{a}, \\
\Lambda_{\mathrm{D}}(t) & =\lambda_{\mathrm{D}}-\lambda_{\mathrm{K}} t, \\
\Lambda_{\mathrm{K}} & =\lambda_{\mathrm{K}} \\
\sigma(\mathbf{x}) & =\sigma_{0}-\lambda_{a} x^{a}+\frac{1}{2} \lambda_{\mathrm{K}} x^{2},
\end{aligned}
$$

where $a^{0}, a^{c}, \lambda^{a b}, \lambda^{a}, \lambda_{\mathrm{D}}, \lambda_{\mathrm{K}}$ and $\sigma_{0}$ are the (constant) parameters of time translations, spatial translations, spatial rotations, Galilean boosts, dilatations, special conformal transformations and central charge transformations, respectively.

The gauge fixing conditions (3.11) imply that after substituting the decompositions (3.9) back into the Galilean invariant one can ignore all terms with the derivative acting on one of the gauge fields. Starting from a general Galilean invariant one thus obtains the field theory of a complex scalar with dilatation weight $w$ and central charge weight $\mathrm{M}$ that is invariant under the following rigid Schrödinger transformations:

$$
\delta \Psi=\xi^{0} \partial_{0} \Psi+\xi^{a} \partial_{a} \Psi+\left(w \Lambda_{\mathrm{D}}+i \mathrm{M} \sigma\right) \Psi
$$

with $\xi^{0}, \xi^{a}, \Lambda_{\mathrm{D}}$ and $\sigma$ given in eq. (3.13).

\subsection{Schrödinger gauge fields}

One may also do the reverse, i.e. derive the Galilean invariant that is dual to a given SFT of a complex scalar. For this purpose, it is convenient to first introduce the gauge fields of Schrödinger gravity and their transformation rules as they follow from gauging the $z=2$ Schrödinger algebra. As explained in appendix A, on top of the independent gauge fields $\tau_{\mu}, e_{\mu}{ }^{a}$ and $m_{\mu}$ introduced above - from now on we omit the superscript "Sch" - we introduce four new Schrödinger gauge fields:

$$
\begin{aligned}
& \omega_{\mu}^{a b} \text { - gauge field of spatial rotations, } \\
& \omega_{\mu}{ }^{a} \text { - gauge field of boosts, } \\
& b_{\mu} \text { - gauge field of dilatations and } \\
& f_{\mu} \text { - gauge field of special conformal transformations, }
\end{aligned}
$$

which are dependent and can be expressed in terms of the independent gauge fields $e_{\mu}^{a}, \tau_{\mu}$ and $m_{\mu}$. The time component of the dilatation gauge field is an exception and remains independent but can be set to zero due to its transformation rule. The transformation rules for all Schrödinger gauge fields are presented in eqs. (A.3) and (A.9). The full details 
including the expressions for all curvatures etc. can be found in [51]. In appendix A we reproduce in our conventions the results of [51] which are necessary for our work.

The spatial components $b_{a}=e_{a}{ }^{\mu} b_{\mu}$ of the dilatation gauge field are dependent and will play the role of torsion terms. Instead, the time component $\tau^{\mu} b_{\mu}$ transforms as a shift under the special conformal transformations. Like in the relativistic case, this symmetry is equivalent to the property that in any Galilean invariant the time-component of the dilatation gauge field $b_{0}=\tau^{\mu} b_{\mu}$ drops out while the spatial components $b_{a}=e_{a}{ }^{\mu} b_{\mu}$, which are dependent, i.e. $b_{a}=b_{a}(e, \tau)$, remain as torsion terms [51]. In contrast, the fate of the central charge gauge field is rather different. This gauge field remains independent and is invariant under the special conformal transformations. It transforms as a shift under Galilean boosts.

With the above information at hand it is straightforward to couple a given SFT of a complex scalar to Schrödinger gravity. In most cases, one just needs to replace all derivatives by covariant derivatives. For the construction of these SFTs and their coupling to Schrödinger gravity, see appendix B and section 5.1, respectively. The corresponding Galilean invariant is then obtained by imposing the gauge-fixing condition

$$
\Psi=1
$$

which fixes both the dilatations and the central charge transformations.

Note that before gauge-fixing $m_{\mu}$ is the gauge field of central charge transformations while after gauge-fixing it is invariant under central charge transformations, i.e. it is not a gauge field anymore but an ordinary vector field. To distinguish between the two cases we will denote the vector field after gauge-fixing with $M_{\mu}$. For the convenience of the reader we summarize here the status of the dilatation gauge field $b_{\mu}$, the central charge gauge field $m_{\mu}$ and the vector field $M_{\mu}$ before and after gauge-fixing:

- before gauge-fixing: $b_{\mu}$ and $m_{\mu}$ are the independent gauge fields of dilatations and central charge transformations, respectively. The time component $b_{0}$ of the dilatation gauge field is the only field that transforms under special conformal transformations. The gauge field $m_{\mu}$ before gauge-fixing is related to the vector field $M_{\mu}$ according to $(3.9 \mathrm{~b})$.

- after gauge-fixing: the time-component $b_{0}$ drops out while the space-components $b_{a}$ which are dependent give rise to torsion. After gauge-fixing there is no difference between $m_{\mu}$ and $M_{\mu}$. They both are vector fields which transform in the same way according to (3.6).

As in the relativistic case, there is a correspondence between Galilean invariants and SFTs of the compensating complex scalar $\Psi$ introduced above. In appendix B we classify all independent SFTs with up to two time derivatives and four spatial derivatives, distinguishing between purely potential terms, see subsection (B.1), and kinetic terms, see subsection (B.2). The lowest order SFT with one time derivative kinetic term is the Schrödinger action, see eq. (B.11). In section 5, we will use these results as a starting point 
to construct, following the procedure developed here, the general Galilean invariants with the same order of derivatives. On top of this, we will also obtain what we refer to as the curvature terms which can not be obtained by SFTs. Note that, unlike in the relativistic case, a general Galilean invariant can have less derivatives than the corresponding SFT. This is a simple consequence of the last equation of (3.9) and is signaled by the presence of the vector field $M_{\mu}$ in the Galilean invariant.

\section{Newton-Cartan gravity}

In the same way that Einstein gravity may be derived from gauging the relativistic Poincaré algebra, the Newton-Cartan formulation of Newtonian gravity can be obtained from gauging the Bargmann algebra, i.e. the centrally extended Galilean algebra [50,61, 62]. In this section we will discuss how to define a consistent set of equations of motion describing Newton-Cartan Gravity (NCG) [6,7] with torsion. We will consider only a special kind of torsion, called 'twistless torsion', which in this context was first discussed in [48]. The torsionless equations can be found in the original literature, see, e.g., [14, 63]. To derive an extension of these equations of motion with torsion, it is very convenient to apply the non-relativistic conformal method developed in section 3. We note that the torsionless equations of motion can be obtained by taking the non-relativistic limit of the Einstein equations $[10,64] .{ }^{11}$ Since the Schrödinger algebra cannot be obtained as the contraction of a relativistic algebra it is not clear a priori whether the NCG equations of motion with torsion we will construct can similarly be obtained as the limit of some relativistic equation of motion. Since there is no NCG action available we will apply the non-relativistic conformal method at the level of the equations of motion. In subsection 4.1 we will first explain the procedure by reproducing the torsionless equations of motion. Then, in subsection 4.2 we will extend this result and construct the NCG equations of motion with twistless torsion.

\subsection{Torsionless NCG}

In this subsection we first discuss the torsionless case. In the absence of torsion the Galilean timelike vielbein field $\left(\tau_{\mu}\right)^{\mathrm{G}}$ satisfies the foliation constraint

$$
\partial_{\mu}\left(\tau_{\nu}\right)^{\mathrm{G}}-\partial_{\nu}\left(\tau_{\mu}\right)^{\mathrm{G}}=0
$$

while the NC equations of motion are given by $[14,50,63]$

$$
\begin{aligned}
\left(\tau^{\mu}\right)^{\mathrm{G}}\left(e^{\nu}{ }_{a}\right)^{\mathrm{G}} \mathcal{R}_{\mu \nu}{ }^{a}(G) & =0, \\
\left(e^{\nu}{ }_{c}\right)^{\mathrm{G}} \mathcal{R}_{\mu \nu}{ }^{c a}(J) & =0,
\end{aligned}
$$

where $\mathcal{R}(G)$ is the NC curvature of Galilean boosts, see eq. (A.11b), and $\mathcal{R}(J)$ is the $\mathrm{NC}$ curvature of spatial rotations, see eq. (A.11a).

Although there is no known action that gives the equations of motion (4.2), we can apply the non-relativistic technique as explained in section 3 to the constraint (4.1) and to

\footnotetext{
${ }^{11}$ We thank Eric Spreen for a discussion on this point.
} 
the equations of motion (4.2). We first consider the constraint (4.1). Upon substitution of the decomposition (3.9) in the constraint (4.1) we obtain

$$
\partial_{\mu}\left(\tau_{\nu}\right)^{\mathrm{Sch}}-\partial_{\nu}\left(\tau_{\mu}\right)^{\mathrm{Sch}}+2 w^{-1} \varphi^{-1}\left(\left(\tau_{\mu}\right)^{\mathrm{Sch}} \partial_{\nu} \varphi-\left(\tau_{\nu}\right)^{\mathrm{Sch}} \partial_{\mu} \varphi\right)=0
$$

where $w$ is the dilatation weight of the compensating scalar $\varphi$. Next, imposing the gaugefixing condition (3.11) in the above equation, we obtain the following constraint:

$$
\partial_{a} \varphi=0
$$

This constraint is invariant under the rigid conformal transformations

$$
\delta \varphi=\xi^{0} \partial_{0} \varphi+\xi^{a} \partial_{a} \varphi+w \Lambda_{\mathrm{D}} \varphi
$$

with $\xi^{0}, \xi^{a}$ and $\Lambda_{\mathrm{D}}$ given in eqs. (3.13).

We next apply the same manipulations to the $\mathrm{NC}$ equation of motion (4.2a). After fixing the dilatation weight to $w=1$, this leads to the following equation for $\varphi:^{12}$

$$
\partial_{0} \partial_{0} \varphi=0
$$

One can show that this equation, together with the constraint equation (4.4), is invariant under the rigid conformal transformations (4.5) for $w=1$. The two equations together define the SFT that underlies the torsionless NCG equations of motion. In the same way curvature terms do not have corresponding SFT actions, there is no SFT corresponding to the equation of motion (4.2b). ${ }^{13}$ Hence, at the moment, we keep this equation as it is.

It is straightforward to recover the torsionless $\mathrm{NC}$ equation of motion (4.2a) from the SFT defined by eqs. (4.4) and (4.6). We first define the covariant derivatives, taken for $w=1$,

$$
\begin{aligned}
& D_{0} \varphi=\tau^{\mu}\left(\partial_{\mu}-w b_{\mu}\right) \varphi, \\
& D_{a} \varphi=e^{\mu}{ }_{a}\left(\partial_{\mu}-w b_{\mu}\right) \varphi .
\end{aligned}
$$

Due to the presence of $b_{0}$ in the time covariant derivative which transforms as a shift under special conformal transformations, we obtain the following second order in time covariant derivative, taken for $w=1$,

$$
D_{0} D_{0} \varphi=\tau^{\mu}\left(\partial_{\mu} D_{0} \varphi-(w-2) b_{\mu} D_{0} \varphi+\omega_{\mu}{ }^{a} D_{a} \varphi+w f_{\mu} \varphi\right)
$$

A remark about the status of the time component $f_{0}=\tau^{\mu} f_{\mu}$ of the gauge field $f_{\mu}$ of special conformal transformations is in order here. When constructing Schrödinger gravity, this gauge field was dependent, see eq. (A.8). In the case of zero torsion, i.e. $b_{a}=0$,

\footnotetext{
${ }^{12}$ This equation was derived together with Jan Rosseel.

${ }^{13}$ If one would try to derive a SFT equation corresponding to the NCG equation (4.2b) one would fail due to the fact that the term $\mathcal{R}_{\mu a}(J)$ decouples from the SFT equation. The situation is similar to that of the Riemann curvature squared in a Poincaré invariant action.
} 
the last two terms in the expression for $f_{0}$ are proportional to the NCG equation of motion (4.2b). Therefore, in this particular case there is no need to add these two terms to the definition of $f_{0}$.

We thus end up with the following two equations describing the coupling of the compensating scalar $\varphi$ of dilatation weight $w=1$ to Schrödinger gravity:

$$
D_{0} D_{0} \varphi=0, \quad D_{a} \varphi=0,
$$

with $f_{0}$ defined by eq. (A.8) with the assumption that eq. (4.2b) is one of the NCG equations of motion. Note that in the torsionless case the two Schrödinger curvature terms in eq. (A.8) effectively reduce to two Galilean curvature terms since $b_{a}=0$ and $b_{0}$ drops out from the final equations of motion due to the invariance under special conformal transformations. Therefore, on-shell we can set the two curvature terms in eq. (A.8) equal to zero. It can be checked that the two equations (4.10) together are invariant under all local Schrödinger transformations for $w=1$. Imposing the gauge-fixing condition

$$
\varphi=1
$$

and using the explicit expressions (A.5)-(A.8) together with eq. (4.2b) the two equations (4.10) reduce to

$$
\tau^{\mu} e^{\nu}{ }_{a} \mathcal{R}_{\mu \nu}{ }^{a}(G)=0, \quad b_{a}=0,
$$

which indeed is the $\mathrm{NC}$ equation of motion (4.2a) together with the statement of zero torsion. Along with our assumption that eq. (4.2b) is valid we thus have reproduced the full set of torsionless NCG equations of motion.

\subsection{Torsional NCG}

In this subsection we consider the general case in which there is nonzero torsion, i.e. $b_{a} \neq 0$. This case is more complicated due to the fact that we a priori neither know the torsional NCG equations of motion nor the corresponding SFT. Of course, we require that the final result, taken for $b_{a}=0$, reduces to the torsionless NCG equations of motion given in the previous subsection.

To derive the extension with torsion, it is easiest to approach the issue from the SFT side. One thing that changes in the torsional case is that the vielbein now satisfies a dilatation-covariant foliation constraint expressing the fact that the torsion is twistless:

$$
\partial_{\mu}\left(\tau_{\nu}\right)^{\mathrm{G}}-\partial_{\nu}\left(\tau_{\mu}\right)^{\mathrm{G}}-2 b_{a}\left(e_{\mu}{ }^{a}\right)^{\mathrm{G}}\left(\tau_{\nu}\right)^{\mathrm{G}}+2 b_{a}\left(e_{\nu}{ }^{a}\right)^{\mathrm{G}}\left(\tau_{\mu}\right)^{\mathrm{G}}=0 .
$$

This constraint is enough to guarantee that the foliation space is a Riemannian manifold. Making a decomposition and gauge-fixing does not lead to the constraint (4.4) anymore as the result of this procedure on (4.13) is automatically vanishing. In the absence of this constraint the equation of motions are no longer invariant under the Galilean symmetries. Equivalently, from the scalar field theory point of view, eq. (4.6) is not invariant under rigid conformal transformations anymore:

$$
\delta\left(\partial_{0} \partial_{0} \varphi\right)=-2 \lambda^{a} \partial_{0} \partial_{a} \varphi
$$


To make equation (4.6) invariant under all rigid Schrödinger transformations, we introduce the second compensating scalar $\chi$ which transforms under rigid conformal transformations as follows:

$$
\delta \chi=\xi^{0} \partial_{0} \chi+\xi^{a} \partial_{a} \chi+\mathrm{M} \sigma,
$$

with $\xi^{0}, \xi^{a}$ and $\sigma$ given in eq. (3.13) and where M is arbitrary. Under Galilean boosts the spatial derivative of $\chi$ transforms with an inhomogeneous term

$$
\delta_{\lambda^{a}} \partial_{a} \chi \sim-\mathrm{M} \lambda_{a} .
$$

This means that at lowest order eq. (4.6) can be made invariant under Galilean boosts by adding a $\chi$-term to that equation. Pursuing this iterative procedure we find the following Schrödinger invariant field equation:

$$
\partial_{0} \partial_{0} \varphi-\frac{2}{\mathrm{M}}\left(\partial_{0} \partial_{a} \varphi\right) \partial_{a} \chi+\frac{1}{\mathrm{M}^{2}}\left(\partial_{a} \partial_{b} \varphi\right) \partial_{a} \chi \partial_{b} \chi=0 .
$$

This is the SFT equation that underlies the torsion extension of the NCG equation of motion (4.2a).

To extract the explicit form of this torsion extension, we promote the rigid Schrödinger symmetry of the SFT equation (4.17) to a local one by coupling the two compensating scalars to Schrödinger gravity. This can be achieved by replacing all ordinary derivatives by Schrödinger-covariant ones: ${ }^{14}$

$$
D_{0} D_{0} \varphi-\frac{2}{\mathrm{M}}\left(D_{0} D_{a} \varphi\right) D_{a} \chi+\frac{1}{\mathrm{M}^{2}}\left(D_{a} D_{b} \varphi\right) D_{a} \chi D_{b} \chi=0 .
$$

We have used here the following definitions of Schrödinger-covariant derivatives (taken for $w=1)$ :

$$
\begin{aligned}
D_{0} D_{a} \varphi & =\tau^{\mu}\left(\partial_{\mu} D_{a} \varphi-(w-1) b_{\mu} D_{a} \varphi-\omega_{\mu a}{ }^{b} D_{b} \varphi\right), \\
D_{a} D_{b} \varphi & =e_{a}^{\mu}\left(\partial_{\mu} D_{b} \varphi-(w-1) b_{\mu} D_{b} \varphi-\omega_{\mu b}{ }^{c} D_{c} \varphi\right) \\
D_{a} \chi & =e_{a}^{\mu}\left(\partial_{\mu} \chi-\mathrm{M} m_{\mu}\right) .
\end{aligned}
$$

Imposing the gauge-fixing conditions

$$
\varphi=1, \quad \chi=0
$$

in eq. (4.18) we obtain the following torsion extension of the NCG equation of motion (4.2a):

$$
\begin{aligned}
\tau^{\mu}\left(\mathcal{R}_{\mu a}{ }^{a}(G)+2 M^{b} \mathcal{R}_{\mu a}{ }^{a} b(J)\right)+M^{b} M^{c} \mathcal{R}_{b a}{ }^{a}{ }_{c}(J)-2 M^{a} K_{a}+\mathcal{D}_{a} b_{a} M^{b} M^{b} & \\
+ & 2 \Omega_{\mu}^{a}\left(-\tau^{\mu} b_{a}+b_{b} e_{b}^{\mu} M^{a}-e_{a}^{\mu} b_{b} M^{b}\right)=0,
\end{aligned}
$$

where $\mathcal{D}$ is the Galilean covariant derivative, see the definition (A.12b), and $K_{a}$ is defined in eq. (A.13a) as the Galilean boost invariant version of $\mathcal{D}_{0} b_{a}$.

\footnotetext{
${ }^{14}$ Note that all covariant derivatives commute such that there is no ambiguity in this procedure, see eq. (5.13).
} 
To obtain the torsional extension of the NCG equation of motion (4.2b) we replace the Galilei curvature $\mathcal{R}(J)$ by the corresponding Schrödinger curvature $R(J)$, see eqs. (A.5a) and (A.10a),

$$
e^{\nu}{ }_{c} R_{\mu \nu}{ }^{c b}(J)=0 \text {. }
$$

Since this equation transforms covariantly under dilatations, it does not lead to a corresponding SFT equation. Note that, upon setting the torsion equal to zero, i.e. $b_{a}=0$, the equations of motion (4.23) and (4.24) reduce to the torsionless equations of motion given in eqs. (4.12) and (4.2b) respectively.

A drawback of the equations of motion (4.23) and (4.24) is that the Galilean invariance is not manifest. One can show that the expression (4.23) can be rewritten in the following manifestly Galilean invariant form:

$$
\hat{\tau}^{\mu} \partial_{\mu} K+K^{a b} K_{a b}-\triangle \Phi-8 \Phi b \cdot b-2 \Phi \mathcal{D} \cdot b-6 b^{a} \mathcal{D}_{a} \Phi=0 .
$$

The dot in equation (4.25) refers to the contraction of the spatial indices, i.e. $b \cdot b \equiv \delta^{a b} b_{a} b_{b}$ and $\mathcal{D} \cdot b \equiv \delta^{a b} \mathcal{D}_{a} b_{b}$. We recall that $\hat{\tau}^{\mu}$ is the Galilean invariant defined by eq. (3.7a). The field $\Phi$ is given by

$$
\Phi \equiv M_{0}+\frac{1}{2} \delta^{a b} M_{a} M_{b}
$$

After gauge-fixing to a frame with constant acceleration, $\Phi$ can be identified with the Newton potential. The Galilean covariant derivatives of $\Phi$ are given by

$$
\mathcal{D}_{a} \Phi=e_{a}^{\mu} \partial_{\mu} \Phi, \quad \triangle \Phi \equiv \delta^{a b} \mathcal{D}_{a} \mathcal{D}_{b} \Phi=e^{\mu a} \partial_{\mu} \mathcal{D}_{a} \Phi-e_{a}^{\mu} \Omega_{\mu}^{a b} \mathcal{D}_{b} \Phi
$$

while $K \equiv \delta^{a b} K_{a b}$ and $K_{a b}$ is defined in eq. (A.13b) as the Galilean boost invariant version of $\mathcal{D}_{a} M_{b}$, a definition that we repeat here for the convenience of the reader

$$
K_{a b}=\mathcal{D}_{a} M_{b}+M_{a} b_{b}+M_{b} b_{a}
$$

Here $\mathcal{D}_{a} M_{b}$ is the Galilean covariant derivative defined in eq. (A.12c). Note that $K_{a b}$ is symmetric as a consequence of the symmetric nature of $\mathcal{D}_{a} M_{b}$, as shown in eq. (A.15), and it can be seen as an extrinsic curvature. One may verify that all the terms appearing in (4.25) are invariant by themselves. The same can be done for the remaining equations of motion (4.24). The two different projections of this equation can be rewritten as

$$
\begin{aligned}
\mathcal{D}^{b} K_{a b}-\mathcal{D}_{a} K+K_{a b} b^{b}-b_{a} K+(d-1) K_{a} & =0, \\
\mathcal{R}_{a c}{ }^{c} b(J)+(d-2)\left(\mathcal{D}_{a} b_{b}+b_{a} b_{b}\right)+\delta_{a b}(\mathcal{D} \cdot b-(d-2) b \cdot b) & =0,
\end{aligned}
$$

where (4.29a) has been obtained by contracting (4.24) with $\hat{\tau}^{\mu}$ and (4.29b) is obtained by contracting (4.24) with $e_{a}^{\mu} .{ }^{15}$ The Galilean covariant derivative of $K_{a b}$ is given by

$$
\mathcal{D}_{a} K_{b c}=e_{a}^{\mu}\left(\partial_{\mu} K_{b c}-\Omega_{\mu b}{ }^{d} K_{d c}-\Omega_{\mu c}{ }^{d} K_{b d}\right)
$$

\footnotetext{
${ }^{15}$ The contraction by $\hat{\tau}^{\mu}$ is necessary in order to make (4.29a) manifestly Galilean invariant by itself.
} 
In order to show that (4.2b) is recovered from eqs. (4.29) in the torsionless limit $b_{a}=0$ one needs to use the identity:

$$
\begin{aligned}
\hat{\tau}^{\mu} \mathcal{R}_{\mu c}{ }^{c}{ }_{a}(J)= & \mathcal{D}^{b} K_{a b}-\mathcal{D}_{a} K+K_{a b} b^{b}-b_{a} K+M^{b} \mathcal{D}_{b} b_{a} \\
& -M_{a}(\mathcal{D} \cdot b+b \cdot b)+b_{a} b_{b} M^{b}+\Omega_{\mu}^{b} e_{b}^{\mu} b_{a}-\delta_{a b} \Omega_{\mu}^{b} e_{c}^{\mu} b^{c} .
\end{aligned}
$$

The equations (4.25) and (4.29) are our final result for the torsional NCG equations of motion. To derive these equations, it was very convenient to have the underlying SFT in mind. Note that equation (4.25) consists of six terms. The term $\triangle \Phi$ yields, after gauge-fixing to an earth-based frame, the Poisson equation of the Newton potential to leading order. Two terms are proportional to the extrinsic curvature and the remaining three terms are all proportional to torsion. Note also that the equation (4.29a) is invariant under time-reversal symmetry since both $K_{a}$ and $K_{a b}$ are odd under that symmetry. As far as we know this general equation has not appeared before in the literature. It would be very interesting to find a non-relativistic situation where these equations of motion should be used, for instance in condensed matter systems, and to construct solutions to these equations.

\section{Hořava-Lifshitz gravity}

In this section we apply the non-relativistic conformal formalism developed in section 3 to SFTs of a compensating complex scalar to obtain a number of (higher-derivative) Galilean invariant actions. To do so, we will first in subsection 5.1 couple the scalar SFTs of appendix B to Schrödinger gravity. In the next subsection 5.2, we will gauge-fix the dilatation and central charge transformations in order to obtain various Galilean invariants. Next, in section 5.3 we will make contact between the higher-derivative Galilean invariants we construct and $z=2$ Hořava-Lifshitz gravity. Our results will be in agreement with those of [13].

\subsection{Scalar coupled Schrödinger gravities}

The aim of this subsection is to classify all possible scalar actions that are invariant under the local Schrödinger transformations. To obtain this result, we start with the SFTs classified in appendix B and couple them to Schrödinger gravity, whose construction is summarized in appendix A. For presentation purposes this procedure is divided into the case of actions with and without time derivatives. We start with the simpler case of purely spatial derivatives in subsubsection 5.1.1. Next, we consider first and second order time-derivatives in subsubsection 5.1.2. In a third subsubsection 5.1.3 we construct locally Schrödinger invariant curvature invariants that do not correspond to a SFT.

The coupling of the SFTs to Schrödinger gravity is obtained by replacing the flat space derivatives $\partial_{0}$ and $\partial_{a}$ by the covariant derivatives $D_{0}=\tau^{\mu}\left(\partial_{\mu}+\ldots\right)$ and $D_{a}=$ $e_{a}^{\mu}\left(\partial_{\mu}+\ldots\right)$ and multiplying the flat space Lagrangian by the determinant $e=\operatorname{det}\left(\tau_{\mu}, e_{\mu}^{a}\right)$. The inverse vielbeins represent the coupling to the Newton-Cartan background and the dots represent the set of gauge fields that need to be added for covariance. This procedure 
can be ambiguous for two distinct reasons. First, because the commutation properties of partial derivatives is in general lost for the covariant derivatives. It turns out that in most cases, at the order at which we are working, the covariant derivatives do commute and this ambiguity does not appear. However, this will not always be the case, see e.g. the discussion around eq. (5.14). The second source of ambiguity is related to the fact that the SFT Lagrangians are defined only up to a total derivative. In order to deal with this ambiguity, we have to make sure that the Lagrangian itself is actually an invariant before we can proceed with covariantizing the SFT. Concretely, as explained in section 2 and appendix B, respectively in the relativistic and non-relativistic cases, this is achieved by imposing the condition (B.2) to the variation of the SFT Lagrangians.

In order to remove possible field redefinitions, we will find it convenient to fix the dilatation weight of the complex scalar field $\Psi$ (and hence also of the real scalar $\varphi$ ) to

$$
w=-\frac{d+2-2 n_{t}-n_{s}}{2},
$$

where $n_{t}$ is the number of time derivatives and $n_{s}$ the number of spatial derivatives in a given term at a given order. We refer the reader to appendix $\mathrm{B}$ for more details.

\subsubsection{The potential terms}

In this section we collect the locally Schrödinger invariant scalar Lagrangians that are zeroth, second and fourth order in spatial derivatives. As follows from the analysis done in appendix B, the potential terms correspond to all the possible inequivalent ways we can act with spatial derivatives on the norm of the scalar field while all indices are contracted.

At the lowest order, with $n_{t}=0$ and $n_{s}=0$, the coupling of (B.5) only amounts to a multiplication by the determinant $e=\operatorname{det}\left(\tau_{\mu}, e_{\mu}^{a}\right)$. Hence, we directly obtain

$$
S^{(0)}=\int d t d^{d} \mathbf{x} e \Lambda_{0} \varphi^{2}
$$

with $w=-\frac{d+2}{2}$ and $\Lambda_{0}$ an arbitrary constant.

With $n_{t}=0, n_{s}=2$ there are only two possible Lagrangians that lead to locally invariant actions. With the dilatation weight fixed to $w=-\frac{d}{2}$ according to (5.1), these are $e D_{a} \varphi D_{a} \varphi$ and $e \varphi \triangle \varphi$ where we denote $\triangle \equiv \delta^{a b} D_{a} D_{b}$. The Schrödinger covariant derivatives are naturally defined from the transformation of the complex field $\Psi=\varphi e^{i \chi}$, see eq. (3.14), and have been given in eqs. (4.8) and (4.20). After coupling to Schrödinger gravity these two Lagrangians differ only by a boundary term:

$$
\varphi \triangle \varphi=-D_{a} \varphi D_{a} \varphi+e^{-1} \partial_{\mu}\left(e \varphi e^{a \mu} D_{a} \varphi\right) .
$$

Hence, we can write a single locally invariant scalar potential action at this order,

$$
S^{(1)}=\int d t d^{d} \mathbf{x} e D_{a} \varphi D_{a} \varphi,
$$

which is the coupled version of (B.6). 
With $n_{t}=0, n_{s}=4$ we can construct invariants by combining $D_{a} \varphi D_{a} \varphi$ and $\varphi \triangle \varphi$ given that in this case the boundary term of (5.3) will have a non-trivial effect. Equivalently, this corresponds to coupling the invariant terms (B.7) to Schrödinger gravity. Explicitly, we obtain

$$
\begin{aligned}
S^{(2)} & =\int d t d^{d} \mathbf{x} e \varphi^{-2}\left(D_{a} \varphi D_{a} \varphi\right)^{2}, \\
S^{(3)} & =\int d t d^{d} \mathbf{x} e \varphi^{-1}\left(D_{a} \varphi D_{a} \varphi\right) \triangle \varphi, \\
S^{(4)} & =\int d t d^{d} \mathbf{x} e(\triangle \varphi)^{2},
\end{aligned}
$$

where local invariance is achieved with the dilatation weight of $\varphi$ fixed to be $w=-\frac{d-2}{2}$. Note that a Lagrangian of the form $e \varphi \triangle^{2} \varphi$ would only differ from the Lagrangian used in $(5.5 \mathrm{c})$ by a boundary term and does not yield a new independent invariant. Also, let us stress that, using the commutation properties of partial derivatives and performing some partial integrations, we could have rewritten the invariant (B.7c) in such a way that after coupling to Schrödinger gravity we would obtain a different invariant;

$$
S^{\left(4^{\prime}\right)}=\int d t d^{d} \mathbf{x} e D^{a} D^{b} \varphi D_{a} D_{b} \varphi
$$

However, due to the relation,

$$
D^{a} D^{b} \varphi D_{a} D_{b} \varphi=(\triangle \varphi)^{2}+D_{a} \varphi D_{b} \varphi R^{a b}(J)+e^{-1} \partial_{\mu}\left[e e_{a}^{\mu}\left(D_{b} \varphi D_{a} D_{b} \varphi-D_{a} \varphi \triangle \varphi\right)\right],
$$

the action (5.6) only differs from the invariant (5.5c) by a curvature term. We will classify such curvature terms later in subsubsection 5.1.3.

Strictly speaking, these are not all possible ways to form independent locally Schrödinger invariant scalar field theories with $n_{t}=0, n_{s}=4$. We could also construct potential terms with the spatial derivative acting on $\chi$. This analysis is done in appendix B.1 and leads to an extra action, the SFT given in (B.9). Although this Lagrangian is invariant in the sense of (2.22) and hence can straightforwardly be coupled to Schrödinger gravity, we show in appendix B.2 that it no longer leads to an independent theory once we consider the SFT's with kinetic terms made of the complex field $\Psi$.

\subsubsection{The kinetic terms}

We now proceed to the coupling of the complex scalar field theories with exactly one and two time-derivatives. At first order in time derivatives the Schrödinger action (B.11), which we repeat here for clarity,

$$
\mathrm{SFT}_{5}: \quad S^{(5)}=\int d t d^{d} \mathbf{x} \Psi^{\star}\left(i \partial_{0}-\frac{1}{2 \mathrm{M}} \partial_{a} \partial_{a}\right) \Psi
$$

describes, up to potential terms, the unique scalar field theory with $n_{t}=1$ that is Schrödinger invariant. The action (5.8) is invariant under the rigid Schrödinger symmetries (3.13) for a complex scalar $\Psi$ of dilatation weight $w=-\frac{d}{2}$ and arbitrary central charge of weight $\mathrm{M}$. 
After coupling the Schrödinger action (5.8) to Schrödinger gravity we obtain

$$
S^{(5)}=\int d t d^{d} \mathbf{x} e \Psi^{\star} \square_{\mathrm{Sch}} \Psi
$$

with $w=-\frac{d}{2}$ and the Schrödinger covariant derivatives given by

$$
\begin{aligned}
\square_{\mathrm{Sch}} \Psi & \equiv\left(i D_{0}-\frac{1}{2 \mathrm{M}} \triangle\right) \Psi \\
D_{0} \Psi & =\tau^{\mu}\left(\partial_{\mu}-w b_{\mu}-i \mathrm{M} m_{\mu}\right) \Psi \\
D_{a} \Psi & =e^{\mu}{ }_{a}\left(\partial_{\mu}-w b_{\mu}-i \mathrm{M} m_{\mu}\right) \Psi \\
\triangle \Psi & =e^{\mu}{ }_{a}\left[\left(\partial_{\mu}-(w-1) b_{\mu}-i \mathrm{M} m_{\mu}\right) D_{a}-\omega_{\mu a}^{b} D_{b}+i \mathrm{M} \omega_{\mu a}\right] \Psi .
\end{aligned}
$$

The local invariance of (5.9) can be easily checked using the transformation rule

$$
\delta\left(\Psi^{\star} \square_{\mathrm{Sch}} \Psi\right)=2(w-1) \Lambda_{D} \Psi^{\star} \square_{\mathrm{Sch}} \Psi-i\left(w+\frac{d}{2}\right) \Lambda_{K} \Psi^{\star} \Psi .
$$

Using the fact that the weight of the determinant $e=\operatorname{det}\left(\tau_{\mu}, e_{\mu}^{a}\right)$ is $d+2$, the action (5.9) is indeed invariant under local Schrödinger transformations for $w=-\frac{d}{2}$. Note that although the action (5.9) is not manifestly real its imaginary part is a boundary term.

We will see that the Galilean invariant corresponding to the Schrödinger action will have inconsistent equations of motion by itself. However, this invariant can be added to the Galilean invariants with two time-derivatives that we will construct below. ${ }^{16}$ Such higher-order Galilean invariants are needed in order to reproduce the kinetic terms of $z=2$ Hořava-Lifshitz gravity, see the actions (5.12a) and (5.12b).

We next consider the Schrödinger scalar theories with two time derivatives, i.e. $n_{t}=2$. To be concrete, we consider the three scalar SFT's classified in appendix B.2, see eqs. (B.14), (B.16) and (B.19) and couple them to Schrödinger gravity. We thus obtain the following three locally Schrödinger invariant actions for a complex scalar field of dilatation weight $w=-\frac{d-2}{2}$ and arbitrary central charge weight $\mathrm{M}$ :

$$
\begin{aligned}
S^{(6)} & =\int d t d^{d} \mathbf{x} e \Psi^{\star} \square_{\mathrm{Sch}}^{2} \Psi \\
S^{(7)} & =\int d t d^{d} \mathbf{x} e\left|\square_{\mathrm{Sch}} \Psi+\frac{1}{\mathrm{M} d}\left(\triangle \Psi-\frac{D_{a} \Psi D_{a} \Psi}{\Psi}\right)\right|^{2}, \\
S^{(8)} & =\int d t d^{d} \mathbf{x} e\left(\Psi^{\star} \Psi\right)^{-1}\left(i \Psi^{\star} D_{0} \Psi-i \Psi D_{0} \Psi^{\star}+\frac{D_{a} \Psi D_{a} \Psi^{\star}}{\mathrm{M}}\right)^{2},
\end{aligned}
$$

where $|\cdot|$ denotes the norm, e.g. $|\Psi|^{2}=\Psi^{\star} \Psi$. There are no ambiguities related to the order of the derivatives in the process of replacing the partial derivatives of the Lagrangians (B.16) and (B.19) by the covariant derivatives of $(5.12 b)$ and (5.12c) due to the identities

$$
\left[D_{a}, D_{b}\right] \Psi=0, \quad\left[D_{0}, D_{a}\right] \Psi=0 .
$$

\footnotetext{
${ }^{16}$ This is similar to the cosmological constant term that, by itself, has an inconsistent equation of motion, but nevertheless can be added to the Einstein-Hilbert action.
} 
However, for the action (5.12a) the order of the covariant derivatives does matter, as can be seen from the non-vanishing of the higher order commutation relation

$$
\left[D_{0}, \triangle\right] \Psi=R(J)_{0 b}^{a b}\left(D_{a}+2 i \mathrm{M} M_{a}\right) \Psi+i \mathrm{M} R(J)_{c b}^{a b} M_{a} M^{c} \Psi
$$

where $R(J)_{\mu \nu}^{a b}$ is the Schrödinger spatial rotation curvature defined in eq. (A.10). Hence, in this particular case, it is the invariance under local Schrödinger transformations that ultimately fixes the correct order in which the covariant derivatives need to appear. We confirm below that, unsurprisingly, $\square_{\text {Sch }}^{2}$ turns out to be the correct combination.

The higher order covariant derivatives are constructed by first determining the transformation rules of the lower-order covariant derivatives acting on $\Psi$. In particular, the higher order covariant derivatives acting on $\Psi$ that occur in eq. (5.12a) are given by

$$
\begin{aligned}
& D_{0}^{2} \Psi=\tau^{\mu}\left[\left(\partial_{\mu}-(w-2) b_{\mu}-i \mathrm{M} m_{\mu}\right) D_{0}+\omega_{\mu}^{a} D_{a}+w f_{\mu}\right] \Psi, \\
& D_{0} \triangle \Psi=\tau^{\mu}\left[\left(\partial_{\mu}-(w-2) b_{\mu}-i \mathrm{M} m_{\mu}\right) \triangle+i \mathrm{M}\left(2 \omega_{\mu}{ }^{a} D_{a}-d f_{\mu}\right)\right] \Psi, \\
& \triangle D_{0} \Psi=e^{\mu}{ }_{a}\left[\left(\partial_{\mu}-(w-3) b_{\mu}-i \mathrm{M} m_{\mu}\right) D_{a} D_{0}-\omega_{\mu a}{ }^{b} D_{b} D_{0}+\omega_{\mu}{ }^{b} D_{a} D_{b}\right. \\
& \left.+(w-1) f_{\mu} D_{a}+i \mathrm{M} \omega_{\mu a} D_{0}\right] \Psi, \\
& \triangle^{2} \Psi=e_{a}^{\mu}\left[\left(\partial_{\mu}-(w-3) b_{\mu}-i \mathrm{M} m_{\mu}\right) D_{a} \triangle-\omega_{\mu a b} D^{b} \triangle\right. \\
& \left.+i \mathrm{M}\left(\omega_{\mu a} \triangle+2 \omega_{\mu}^{b} D_{a} D_{b}-(d+2) f_{\mu} D_{a}\right)\right] \Psi .
\end{aligned}
$$

A few remarks are in order. The invariance of the action (5.12a) under Schrödinger symmetries can be confirmed from the transformation rule

$$
\delta\left(\Psi^{\star} \square_{\mathrm{Sch}}^{2} \Psi\right)=(w-4) \Lambda_{D} \Psi^{\star} \square_{\mathrm{Sch}}^{2} \Psi-i(2 w-2+d) \Lambda_{K} \Psi^{\star} \square_{\mathrm{Sch}} \Psi
$$

where the last term of (5.16) drops given that the dilatation weight is fixed to $w=-\frac{d-2}{2}$.

It is instructive to consider the variation of the second order covariant time derivative

$$
\delta\left(D_{0}^{2} \Psi\right)=\left[(w-4) \Lambda_{D}+i \mathrm{M} \sigma\right] D_{0}^{2} \Psi-2 \lambda^{a} D_{a} D_{0} \Psi-2(w-1) \Lambda_{K} D_{0} \Psi .
$$

As we confirmed, the action (5.12a) contains precisely the terms needed to compensate for the variation (5.17). Nevertheless, we note that the last term in (5.17) could also vanish with a dilatation weight $w=1$. This fact has actually been used in section 4 in the context of the invariance of the Newton-Cartan equations of motion which has been achieved precisely at $w=1$ using an additional constraint.

The invariant action (5.12b) can be seen as the non-relativistic analogue to the gravity coupled version of the relativistic $\mathrm{CFT}_{3}$ given in eq. (2.27). The fact that, unlike in the relativistic case, we have another invariant (5.12c), on top of (5.12a) and (5.12b), is specific to the fact that we are considering a complex scalar field. This is apparent from the classification in appendix B.2. This additional invariant can be obtained from the partially 
integrated Schrödinger action (5.9). We indicate it with a prime to distinguish it from the original Schrödinger action:

$$
S^{\left(5^{\prime}\right)} \equiv \int d t d^{d} \mathbf{x} e\left(i \Psi^{\star} D_{0} \Psi-i \Psi D_{0} \Psi^{\star}+\frac{1}{\mathrm{M}} D_{a} \Psi^{\star} D_{a} \Psi\right) .
$$

This elucidates the relation between the action (5.12c) and the Schrödinger action, namely, the Lagrangian corresponding to the action (5.12c) is nothing else than the square of the Schrödinger Lagrangian (5.18). Note that the Lagrangian (5.18) is manifestly real. An important difference between the variation of the Lagrangians in equations (5.9) and (5.18) is that the special conformal transformations fix $w=-\frac{d}{2}$ in the former case, see eq. (5.11), whereas they do not fix the dilatation weight in the second case. Explicitly one finds $\delta L_{\left(5^{\prime}\right)}=2(w-1) \Lambda_{D} L_{\left(5^{\prime}\right)}$, with

$$
L_{\left(5^{\prime}\right)} \equiv e^{-1} \mathcal{L}_{\left(5^{\prime}\right)}=i \Psi^{\star} D_{0} \Psi-i \Psi D_{0} \Psi^{\star}+\frac{1}{\mathrm{M}} D_{a} \Psi^{\star} D_{a} \Psi .
$$

As a consequence, the Lagrangian (5.18) can be squared, or taken to any higher power, to construct invariant actions at higher orders with $w \neq-\frac{d}{2}$. An example of such a construction will follow below. In subsection 5.2, we will explicitly see that after gauge fixing the coupled scalar field theories (5.12a), (5.12b) and (5.12c) lead to three different Galilean invariants.

Following the classification of appendix B.2 we finally consider the SFT's that mix one time and two spatial derivatives. There are two such theories, see eqs. (B.22a) and (B.22b). They are Schrödinger invariant for a complex scalar field of dilatation weight $w=-\frac{d-2}{2}$, as follows from the general expression (5.1). They can be coupled to Schrödinger gravity in a straightforward way

$$
\begin{aligned}
S^{(9)} & =\int d t d^{d} \mathbf{x} e \varphi^{-2} L_{\left(5^{\prime}\right)} D_{a} \varphi D_{a} \varphi, \\
S^{(10)} & =\int d t d^{d} \mathbf{x} e \varphi^{-1} L_{\left(5^{\prime}\right)} \triangle \varphi .
\end{aligned}
$$

The invariance of actions (5.20a) and (5.20b) follows from the fact that they are combinations of the Lagrangian (5.19) together with the building blocks (5.5) that were used in the construction of the potential terms. We will see that although from the scalar field point of view these theories have a time derivative, after gauge fixing in subsection 5.2, the invariants (5.20a) and (5.20b) will actually lead to Galilean potential terms containing only spatial derivatives.

\subsubsection{The curvature terms}

As mentioned in section 2, in the relativistic case, there exist Poincaré invariants that do not arise from the coupling of scalar CFT's to conformal gravity. Here, in the same way, we expect that there exist locally Schrödinger invariants that are not related to any of the SFT's constructed in appendix B. Indeed, noticing that the Schödinger spatial rotation curvature $R_{a b c d}(J)$, defined in eq. (A.10), only transforms under rotations and dilatations 


\begin{tabular}{|c|cccc|}
\hline$w=-\frac{d-2}{2}$ & $S^{(12)}$ & $S^{(13)}$ & $S^{(14)}$ & $S^{(15)}$ \\
\hline$e^{-1} \mathcal{L}$ & $R(J)^{2} \varphi^{2}$ & $D_{a} \varphi D_{a} \varphi R(J)$ & $\varphi \triangle \varphi R(J)$ & $L_{\left(5^{\prime}\right)} R(J) \varphi^{2}$ \\
\hline
\end{tabular}

Table 1. This table indicates the four curvature invariants $S^{(12)}-S^{(15)}$ that can be constructed using the contracted spatial rotation curvature $R(J)$.

\begin{tabular}{|c|ccc|}
\hline$w=-\frac{d-2}{2}$ & $S^{(16)}$ & $S^{(17)}$ & $S^{(18)}$ \\
\hline$e^{-1} \mathcal{L}$ & $D_{a} \varphi D_{b} \varphi R^{a b}(J)$ & $\varphi^{2} R_{a b}(J) R^{a b}(J)$ & $\varphi^{2} R_{a b c d}(J) R^{a b c d}(J)$ \\
\hline
\end{tabular}

Table 2. This table indicates the three additional curvature invariants $S^{(16)}-S^{(18)}$ that can be constructed using the spatial rotation curvature $R^{a b}(J)$ or $R^{a b c d}(J)$.

(with weight 2) and is invariant under the rest of the Schrödinger symmetries, it is clear that we can construct invariants out it. On the other hand, due to the fact that $R_{a b c d}(J)=0$ in the flat space limit, it is also obvious that these invariants cannot arise from a scalar field theory in flat space.

In this section we build all possible locally Schrödinger invariant actions for the compensating scalar $\Psi$, with fixed dilatation weights $w=-\frac{d}{2}$ and $w=-\frac{d-2}{2}$, that are built out of curvatures of Schrödinger gravity. As it turns out, only $\varphi$, the norm of $\Psi$, will actually appear in the invariants. In the construction of Schrödinger gravity most curvatures are set to zero as constraints in order to solve for the dependent gauge fields (A.5) and (A.8). The only non-vanishing curvatures remaining are some components of the Schrödinger spatial rotation, Galilean boost and special conformal transformation curvatures, see [51] for the full details. However, it can be seen that all the invariants that can be built using the Galilean boost and special conformal transformation curvatures as well as $R_{0 a b c}(J)$ do necessarily break time-reversal symmetry. This is the reason why all the invariants we consider below are constructed only out of $R_{a b c d}(J)$.

We start by enumerating the invariants that involve $R(J) \equiv R(J)_{a b}{ }^{a b}$. At second order in spatial derivatives we can form the unique invariant

$$
S^{(11)}=\int d t d^{d} \mathbf{x} e R(J) \varphi^{2}, \quad w=-\frac{d}{2} .
$$

At higher order, it is possible to combine $R(J)$ with itself, or together with $D_{a} \varphi D_{a} \varphi, \triangle \varphi$ and $L_{\left(5^{\prime}\right)}$ to form new invariants. We recall that $L_{\left(5^{\prime}\right)}$ has been defined in eq. (5.19). We summarize the invariants constructed in this way by giving the corresponding Lagrangians in table 1.

Of course, we can also construct additional invariants using other contractions of the Schrödinger rotation curvature tensor $R_{a b c d}(J)$. This allows us to write three more invariants that we summarize in table 2 .

Note that a Lagrangian of the form $\varphi D_{a} D_{b} \varphi R^{a b}(J)$ does not lead to a new independent invariant. It only differs from a combination of the invariant actions $S^{13}, S^{14}$ and $S^{16}$ by 
a boundary term due to the identity

$$
\begin{aligned}
\varphi D_{a} D_{b} \varphi R^{a b}(J)= & \frac{1}{2} \varphi \triangle \varphi R(J)-D_{a} \varphi D_{b} \varphi R^{a b}(J)+\frac{1}{2} D_{a} \varphi D_{a} \varphi R(J) \\
& +e^{-1} \partial_{\mu}\left[e \varphi e_{a}^{\mu} D_{b} \varphi\left(R^{a b}(J)-\frac{1}{2} \delta^{a b} R(J)\right)\right] .
\end{aligned}
$$

These are all locally Schrödinger invariant actions built out of the Schrödinger curvatures at this order.

\subsection{Gauge fixing}

The scalar actions we have constructed so far are all locally invariant under Schrödinger symmetries. In this subsection we impose the gauge-fixing condition (3.15) on the invariant actions constructed in section 5.1 to obtain the corresponding Galilean invariants. To do so, we extensively make use of the formulas of appendix A in order to rewrite all Schrödinger dependent gauge fields in terms of purely Galilean quantities. At the end of this section we summarize in the tables 3 and 4 the independent Galilean invariants that correspond to the actions $S^{(0-10)}$ and $S^{(11-18)}$, respectively.

We start by gauge fixing the invariant action $S^{(0)}$ which, upon fixing $\varphi=1$, trivially leads to a cosmological constant

$$
\mathrm{Gal}_{0}: e^{-1} \mathcal{L}_{\mathrm{G}}=\Lambda_{0}
$$

Next we gauge-fix the invariant actions $S^{(1-4)}$ that are related to potential terms. These actions will contain the same number of derivatives before and after gauge fixing. The action $S^{(1)}$, see eq. (5.4), is invariant for a scalar field of weight $w=-\frac{d}{2}$. Using the definition of the covariant derivative (4.8), we obtain after gauge fixing the following Galilean invariant:

$$
\mathrm{Gal}_{1}: e^{-1} \mathcal{L}_{\mathrm{G}}=w^{2} b \cdot b,
$$

where we recall that the dot refers to the contraction of the spatial indices. In the same way, we get for the higher-order potential terms,

$$
\begin{aligned}
& \mathrm{Gal}_{2}: e^{-1} \mathcal{L}_{\mathrm{G}}=w^{4}(b \cdot b)^{2}, \\
& \mathrm{Gal}_{3}: e^{-1} \mathcal{L}_{\mathrm{G}}=-w^{3}(b \cdot b)(\mathcal{D} \cdot b+w b \cdot b), \\
& \mathrm{Gal}_{4}: e^{-1} \mathcal{L}_{\mathrm{G}}=w^{2}(\mathcal{D} \cdot b+w b \cdot b)^{2},
\end{aligned}
$$

with $w=-\frac{d-2}{2}$, and where the curly $\mathcal{D}$ is the Galilean covariant derivative, see eq. (A.12b) for its definition. Note that although $\mathcal{D} \cdot b$ is by itself a Galilean invariant, it does not lead to an independent invariant at second order due to the fact that $e(\mathcal{D} \cdot b+2 b \cdot b)$ is exactly a boundary term, see eq. (A.14). This is consistent with what has been found from the local SFT point of view.

We continue with the prime example of a one-derivative kinetic term which is the Schrödinger action (5.8) that has been coupled to Schrödinger gravity in eq. (5.9). By 
inserting the explicit form of the dependent gauge fields in (5.9) and applying the gauge fixing condition $\Psi=1$, we obtain the following Lagrangian

$$
\mathrm{Gal}_{5}: \quad e^{-1} \mathcal{L}_{\mathrm{G}}=\mathrm{M} \Phi+\frac{1}{2 \mathrm{M}} w^{2} b \cdot b,
$$

with $w=-\frac{d}{2}$ and where $\Phi$ is defined in eq. (4.26). After gauge-fixing to a frame with constant acceleration, the field $\Phi$ can be identified with the Newton potential. Note that the term $b_{0}$ has completely disappeared from the final result as expected from the invariance under special conformal transformations.

It turns out that by itself the Galilean invariant that is dual to the Schrödinger action (5.8) has inconsistent equations of motion. ${ }^{17}$ However, it can consistently be added to the Hořava-Lifshitz action as we will discuss in section 5.3. Furthermore, it also shows up in the construction of the equations of motion for Newton-Cartan gravity in section 4 . As we saw above, $b \cdot b$ is an independent invariant which is generated by $S^{1}$, see eq. (5.24). Hence, $\Phi$ is the only independent Galilean invariant produced by the Schrödinger action.

We just showed that the Schrödinger action is unable to produce a kinetic term for the gravitational theory obtained after gauge fixing. This situation changes if we consider SFT's which are second order in the time derivatives. In that case a kinetic term can be generated. The coupled complex scalar field theories $S^{(6-8)}$ given in eqs. (5.12) are the only ones that are Schrödinger invariant with second order time derivatives, see appendix B. We will see that after gauge fixing the invariants $S^{(6,7)}$ will produce kinetic terms whereas the Galilean invariant produced by $S^{(8)}$ will not. Instead, the presence of this last term will be related to whether the field $\Phi$ can be integrated out from the final theory or not. We will come back to this point in section 5.3.

Upon gauge fixing $\Psi=1$ in the actions (5.12), using $w=-\frac{d-2}{2}$ and after removing the boundary terms, we produce the following Galilean invariants,

$$
\begin{aligned}
\mathrm{Gal}_{6}: \quad e^{-1} \mathcal{L}_{\mathrm{G}}= & \frac{1}{d} K^{a b} K_{a b}-\frac{(w+1)}{2 d}\left(K^{a}{ }_{a}\right)^{2}+2 w \Phi\left(\left(1+d^{-1}\right) \mathcal{D} \cdot b+w b \cdot b\right) \\
& +\mathrm{M}^{2}\left(\Phi-\frac{w}{2 \mathrm{M}^{2}}(\mathcal{D} \cdot b+w b \cdot b)\right)^{2} \\
\mathrm{Gal}_{7}: \quad e^{-1} \mathcal{L}_{\mathrm{G}}= & \frac{w^{2}}{d^{2}}\left(K^{a}{ }_{a}\right)^{2}+\mathrm{M}^{2}\left(\Phi-\frac{w^{2}}{d \mathrm{M}^{2}}(\mathcal{D} \cdot b+(w+1) b \cdot b)\right)^{2} \\
\mathrm{Gal}_{8}: \quad e^{-1} \mathcal{L}_{\mathrm{G}}= & \mathrm{M}^{2}\left(2 \Phi+\frac{w^{2}}{\mathrm{M}^{2}} b \cdot b\right)^{2}
\end{aligned}
$$

where $K_{a b}$ has been defined in eq. (4.28). In section 5.3 we will show that the terms $K^{a b} K_{a b}$ and $\left(K_{a}^{a}\right)^{2}$ correspond to the kinetic terms of $z=2$ Hořava-Lifshitz Gravity. ${ }^{18}$ As expected, the $b_{0}$ contributions have canceled out in the final results as a consequence

\footnotetext{
${ }^{17}$ This may be compared to the relativistic case where the cosmological constant by itself has an inconsistent equation of motion but it may be added in a consistent way to the Einstein-Hilbert action where it leads to a modification of the Einstein equations. See also footnote 16.

${ }^{18} K_{a b}$ can be seen to correspond to a kinetic term due to the presence of the Galilean boost connection $\Omega_{\mu}^{a}$, see equations (A.7) and (A.12c) of appendix A.
} 


\begin{tabular}{|c|ccccccccccc|}
\hline SFT & $(0)$ & $(1)$ & $(2)$ & $(3)$ & $(4)$ & $(5)$ & $(6)$ & $(7)$ & $(8)$ & $(9)$ & $(10)$ \\
\hline Gal. Inv. & $\Lambda_{0}$ & $b^{2}$ & $b^{4}$ & $b^{2} \mathcal{D} \cdot b$ & $(\mathcal{D} \cdot b)^{2}$ & $\Phi$ & $K^{a b} K_{a b}$ & $\left(K^{a}{ }_{a}\right)^{2}$ & $\Phi^{2}$ & $\Phi b^{2}$ & $\Phi \mathcal{D} \cdot b$ \\
\hline
\end{tabular}

Table 3. This table indicates the independent Galilean invariants that are produced by their corresponding SFT's. By (0) in the first row we mean $S^{(0)}$, etc.

of the invariance under special conformal transformation. After computing the Galilean invariants $\mathrm{Gal}_{9}$ and $\mathrm{Gal}_{10}$, see below, we will conclude that $K^{a b} K_{a b},\left(K^{a}{ }_{a}\right)^{2}$ and $\Phi^{2}$ are the three independent Galilean invariants that are generated by the actions $S^{(6-8)}$.

The invariant actions $S^{(9-10)}$, given by eqs. (5.20), are first order in time derivative and second order in spatial derivatives. After gauge-fixing they only preserve their number of spatial derivatives. This is signaled by the presence of the vector field $M_{\mu}$ in the definition of $\Phi$. To be concrete, we obtain in this case, for $w=-\frac{d-2}{2}$,

$$
\begin{aligned}
\mathrm{Gal}_{9}: & e^{-1} \mathcal{L}_{\mathrm{G}}=\mathrm{M} w^{2}(b \cdot b)\left(2 \Phi+\frac{w^{2}}{\mathrm{M}^{2}} b \cdot b\right) \\
\mathrm{Gal}_{10}: & e^{-1} \mathcal{L}_{\mathrm{G}}=-\mathrm{M} w(\mathcal{D} \cdot b+w b \cdot b)\left(2 \Phi+\frac{w^{2}}{\mathrm{M}^{2}} b \cdot b\right)
\end{aligned}
$$

producing the new independent Galilean invariants $\Phi b \cdot b$ and $\Phi \mathcal{D} \cdot b$.

For the convenience of the reader, we summarize in table 3 the independent Galilean invariants that we derived so far for each of the corresponding scalar SFT's after coupling them to Schrödinger gravity and performing a gauge fixing.

Finally, we consider the Galilean invariants that are associated with the actions built out of the Schrödinger rotation curvature tensor $R(J)_{a b c d}$ in subsubsection 5.1.3. For this purpose, we express $R(J)_{a b c d}$ in terms of the Galilean rotation curvature tensor $\mathcal{R}_{a b c d}(J)$, see its definition (A.11). These two curvatures are related by

$$
\begin{aligned}
R(J)_{a b c d}= & \mathcal{R}_{a b c d}(J)+\mathcal{D}_{a} b_{[d} \delta_{c] b}-\mathcal{D}_{b} b_{[d} \delta_{c] a}+\mathcal{D}_{d} b_{[a} \delta_{b] c}-\mathcal{D}_{c} b_{[a} \delta_{b] d} \\
& +2\left(b_{b} b_{[c} \delta_{d] a}-b_{a} b_{[c} \delta_{d] b}\right)+(b \cdot b)\left(\delta_{a c} \delta_{b d}-\delta_{a d} \delta_{b c}\right)
\end{aligned}
$$

Using expression (5.31), we can rewrite the action $S^{(11)}$, see eq. (5.21), after gauge-fixing as follows:

$$
\operatorname{Gal}_{11}: \quad e^{-1} \mathcal{L}_{\mathrm{G}}=\mathcal{R}+(d-1)(2 \mathcal{D} \cdot b-(d-2) b \cdot b) .
$$

The Galilean rotation curvature, $\mathcal{R}=\mathcal{R}^{a b}{ }_{a b}(J)$, is the only newly generated Galilean invariant. We recall that $\mathcal{D} \cdot b \propto b \cdot b$ up to a boundary term.

Similarly, from expression (5.31) and the Galilean invariants that we already found, see table 3 , it can be seen that the actions $S^{(12-18)}$ produce Galilean invariants for which only the $\mathcal{R}_{a b c d}$ contribution is leading to a new independent Galilean invariant. The remaining terms on the right hand side of eq. (5.31) do not produce anything new. For simplicity, we only summarize the independent piece of the results in table 4.

This finishes our discussion of the non-relativistic conformal method applied to the Schrödinger invariant actions to obtain a number of Galilean invariants. These invariants 


\begin{tabular}{|c|cccccccc|}
\hline Curv. terms & $(11)$ & $(12)$ & $(13)$ & $(14)$ & $(15)$ & $(16)$ & $(17)$ & $(18)$ \\
\hline Gal. Inv. & $\mathcal{R}$ & $\mathcal{R}^{2}$ & $b^{2} \mathcal{R}$ & $(\mathcal{D} \cdot b) \mathcal{R}$ & $\Phi \mathcal{R}$ & $b_{a} b_{b} \mathcal{R}^{a b}$ & $\mathcal{R}^{a b} \mathcal{R}_{a b}$ & $\mathcal{R}^{a b c d} \mathcal{R}_{a b c d}$ \\
\hline
\end{tabular}

Table 4. This table indicates the Galilean invariants that are produced by the curvature terms discussed in subsubsection 4.1.3. The (11) in the first row refers to the corresponding invariant action $S^{(11)}$, etc.

appear naturally in the $z=2$ Hořava-Lifshitz gravity action which we discuss in the following section 5.3.

\subsection{Identification with Hořava-Lifshitz gravity}

In this section we closely follow [13] where it was first shown that, by making the NC geometry dynamical, HL gravity is reproduced from an action containing a collection of higher-derivative Galilean invariants. These are precisely the same invariants that we have derived in subsection 5.2 using the non-relativistic conformal method. For the convenience of the reader, and to make this work self-contained, we briefly repeat some of the arguments of [13] using our own notation.

To start with, the most general action that we can construct out of the Galilean invariants obtained in section 5.2 is of the form

$$
S=\frac{1}{\kappa^{2}} \int d t d^{d} \mathbf{x} e\left(K_{a b} K^{a b}-\lambda\left(K_{a}^{a}\right)^{2}+\mathcal{V}\right),
$$

where $\lambda$ is a parameter and where the potential $\mathcal{V}$ contains any combination of the potential terms summarized in table 3 and table 4 , namely

$$
\begin{aligned}
\mathcal{V}= & \Lambda_{0}+\lambda_{1} \Phi+\lambda_{2} \Phi^{2}+\lambda_{3} b^{2}+\lambda_{4} b^{4}+\lambda_{5} b^{2} \mathcal{D} \cdot b+\lambda_{6}(\mathcal{D} \cdot b)^{2}+\lambda_{7} \Phi b^{2}+\lambda_{8} \Phi \mathcal{D} \cdot b \\
& +\lambda_{9} \mathcal{R}+\lambda_{10} \mathcal{R}^{2}+\lambda_{11} b^{2} \mathcal{R}+\lambda_{12}(\mathcal{D} \cdot b) \mathcal{R}+\lambda_{13} \Phi \mathcal{R} \\
& +\lambda_{14} b_{a} b_{b} \mathcal{R}^{a b}+\lambda_{15} \mathcal{R}^{a b} \mathcal{R}_{a b}+\lambda_{16} \mathcal{R}^{a b c d} \mathcal{R}_{a b c d}
\end{aligned}
$$

for arbitrary coefficients $\lambda_{1}, \ldots, \lambda_{16}$ and $\Lambda_{0}$. Note that if the coefficient $\lambda_{2}$, in front of the $\Phi^{2}$ term, is non-vanishing then the field $\Phi$ can be integrated out. On the other hand, if $\lambda_{2}=0$ the equations of motion for $\Phi$ lead to a constraint equation.

We first identify our kinetic terms with the standard HL kinetic terms [8, 9]. In order to make the connection between our formalism and the one usually used in HL gravity we first rewrite the kinetic terms in terms of the spatial degenerate metric $h_{\mu \nu} \equiv \delta_{a b} e_{\mu}^{a} e_{\nu}^{b}$ and remove all the occurrences of the spatial vielbein $e_{\mu}^{a}$. Using the definition of $K_{a b}$ given by eq. (4.28) we find that

$$
K_{a b}=e_{a}^{\mu} e_{b}^{\nu}\left(\frac{1}{2} \mathcal{L}_{\tau}\left(h_{\mu \nu}\right)+\frac{1}{2} \nabla_{\mu}\left(h_{\nu}{ }^{\rho} M_{\rho}\right)+\frac{1}{2} \nabla_{\nu}\left(h_{\mu}{ }^{\rho} M_{\rho}\right)+2 M_{(\mu} b_{\nu)}\right),
$$

where

$$
\begin{aligned}
\mathcal{L}_{\tau}\left(h_{\mu \nu}\right) & =\tau^{\rho} \partial_{\rho} h_{\mu \nu}+h_{\mu \rho} \partial_{\nu} \tau^{\rho}+h_{\rho \nu} \partial_{\mu} \tau^{\rho} \\
\nabla_{\mu} M_{\nu} & =\partial_{\mu} M_{\nu}-\Gamma_{\mu \nu}^{\rho} M_{\rho} \\
\Gamma_{\mu \nu}^{\rho} & =\frac{1}{2} h^{\rho \sigma}\left(\partial_{\mu} h_{\nu \sigma}+\partial_{\nu} h_{\mu \sigma}+\partial_{\sigma} h_{\mu \nu}\right)
\end{aligned}
$$


with $h_{\mu}{ }^{\nu}$ the spatial projector $h_{\mu}{ }^{\nu}=h_{\mu \rho} h^{\nu \rho}$. Note that the expression (5.38) is the only part of the Christoffel connection that is appearing due to the overall contraction with $e_{a}^{\mu} e_{b}^{\nu}$ in (5.35). Here, this $\Gamma_{\mu \nu}^{\rho}$ is merely used to obtain a rewriting of our kinetic terms in a purely metric formulation and should not be seen as a full connection. From the expression (5.35), it can then be checked that using a foliated ADM decomposition ${ }^{19}$ with lapse and shift variables $N$ and $N_{i}$, respectively we have

$$
\tau_{\mu}=\left(\begin{array}{c}
N \\
0
\end{array}\right), \quad h_{\mu \nu}=\left(\begin{array}{cc}
0 & 0 \\
0 & \gamma_{i j}
\end{array}\right), \quad M_{\mu}=\left(\begin{array}{c}
N M_{0} \\
-N_{i} / N
\end{array}\right),
$$

we can rewrite our kinetic terms in the form $K^{a b} K_{a b}=K^{i j} K_{i j}$ and $K_{a}^{a}=\gamma^{i j} K_{i j}$ with

$$
K_{i j}=\frac{1}{2} N^{-1}\left(\partial_{t} \gamma_{i j}-\nabla_{i} N_{j}-\nabla_{j} N_{i}\right),
$$

where $\nabla_{i}$ contains the standard Christoffel connection with respect to the invertible metric $\gamma_{i j}$. This is exactly the extrinsic curvature of HL gravity, and hence this shows the identification of the kinetic terms. For a more detailed dictionary between the Newton-Cartan and HL formalisms we refer the reader to [13].

The lapse field $N$ is the gauge field associated with time reparametrizations. In projectable HL gravity this gauge field is restricted to be a projectable function on the spacetime foliation to preserve the time foliation, i.e. $\partial_{a} N=0$. Using the expression (A.5c) for $b_{a}$ it follows that this corresponds to the zero torsion case where $b_{a}=0$. This condition reduces the number of potential terms in the theory.

The HL action (5.33) that we obtain differs from the one obtained in [13] by the fact that it is not restricted to $d=2$. We obtain the result (5.33) for arbitrary $d>0 .{ }^{20}$ However, in the present setup, our analysis is restricted to $z=2$ instead. Moreover, because we consider SFTs only up to second order in time derivatives and fourth order in spatial derivatives, we reproduce the HL gravity potential considered e.g. in [65-67] only up to the higher order derivative terms containing more than four spatial derivatives.

\section{Conclusions}

The relativistic conformal method has turned out to be very useful in many supergravity constructions. In this paper we have developed a non-relativistic analogue of this formalism where the conformal algebra has been replaced by the smallest conformal extension of the Bargmann algebra, i.e. the $z=2$ Schrödinger algebra. The method guarantees that for each $z=2$ SFT one can construct a Galilean invariant. In this way one has a systematic way of constructing all Galilean invariants of a given type except for the so-called curvature terms that do not correspond to a SFT.

In this paper we applied the non-relativistic conformal method to the SFT's with upto two time and four spatial derivatives. In this way we obtained a number of higherderivative Galilean invariants that could be identified with $z=2$ Hořava-Lifshitz gravity

\footnotetext{
${ }^{19}$ We split the $\mu$ index into the coordinates $t$ and $x^{i}$.

${ }^{20}$ As mentioned in appendix $\mathrm{B}$, the case $d=2$ is included in our analysis although, by fixing the dilatation weight of the compensating scalar according to (5.1) for simplicity, we do not show this explicitly.
} 
thereby reproducing the results of [13]. We expect that the non-relativistic conformal method will come to its full power once one wishes to study more complicated cases such as the supersymmetric extension of HL gravity. A first step in this direction has been taken in [68] following on the development of Newton-Cartan supergravity [64, 69]. Moreover, the classification of the higher order SFT's performed in appendix B is interesting in its own right and, in a different context, it could potentially be useful for other applications.

We also applied the non-relativistic conformal method to construct the equations of motion of curved space NC gravity with torsion, a result that, as far as we know, has not appeared before in the literature. In this case, it was clearly an advantage to first extend the underlying $z=2$ SFT to the case with non-zero torsion instead of doing this straightaway in the NC equations of motion themselves. A peculiar feature of this construction is that we had to work with a SFT that was only defined in terms of equations of motion that, with the given number of fields, could not be integrated to an action. This reflects the property of $\mathrm{NC}$ gravity itself which is only formulated in terms of equations of motion without a clear underlying action principle. This is in contrast to the case of HL gravity considered in this paper which has an underlying action principle.

The formalism we developed in this work is naturally formulated in arbitrary dimensions. The generalization to values $z \neq 2$ of the dynamical exponent is less obvious. A necessary ingredient in achieving this is to first construct Schrödinger gravity for $z \neq 2$. Fortunately, this has been already done in [51]. It would be interesting to continue this program and construct the relevant SFT's. We expect that the analogy with the relativistic conformal programme will be less obvious due to the absence of the special conformal transformations for $z \neq 2$.

Finally, we note that the Schrödinger symmetries cannot be obtained as the nonrelativistic limit of the conformal symmetries. Instead, one obtains the Galilean conformal symmetries that have also occurred in studies of non-relativistic holography [23]. These symmetries are truly conformal in the sense that they do not allow a mass parameter. It would be interesting to see whether the non-relativistic conformal method can be extended to these Galilean conformal symmetries as well.

\section{Acknowledgments}

We are grateful to Mehmet Özkan for his many clarifications on the conformal method. We also thank Jan Rosseel, Charles Melby-Thompson and Thomas Zojer for useful discussions on non-relativistic gravity. We thank Quim Gomis, Jelle Hartong and Niels Obers for comments about an earlier version of this manuscript. HA was supported by the Dutch stichting voor Fundamenteel Onderzoek der Materie (FOM). His work is supported in part by Iranian National Science Fundation (INSF). BR is supported by the Dutch stichting voor Fundamenteel Onderzoek der Materie (FOM). AM and PP were supported by Erasmus Mundus NAMASTE India-EU Grants. Some of the calculations have been performed using the software package Cadabra [70]. 


\begin{tabular}{|lllllll|}
\hline$H$ & $P_{a}$ & $G_{a}$ & $J_{a b}$ & $D$ & $K$ & $N$ \\
\hline$\xi^{0}$ & $\xi^{a}$ & $\Lambda^{a}$ & $\Lambda^{a b}$ & $\Lambda_{D}$ & $\Lambda_{K}$ & $\sigma$ \\
$\tau_{\mu}$ & $e_{\mu}{ }^{a}$ & $\omega_{\mu}{ }^{a}$ & $\omega_{\mu}{ }^{a b}$ & $b_{\mu}$ & $f_{\mu}$ & $m_{\mu}$ \\
\hline
\end{tabular}

Table 5. This table indicates the generators, parameters and gauge fields of the $z=2$ Schrödinger algebra.

\section{A Schrödinger gravity}

In this appendix we collect a few formulae related to Schrödinger gravity which is obtained by gauging the $z=2$ Schrödinger algebra [51]. In the main text, we will find it useful to express the Schrödinger quantities in terms of the gauge fields and curvatures of the Bargmann algebra. For this purpose, it is convenient to relate in this appendix the Schrödinger gauge fields and curvatures to the Bargmann gauge fields and curvatures describing the Newton-Cartan geometry [50].

The $z=2$ Schrödinger algebra in $d+1$ dimensions reads

$$
\begin{aligned}
& {\left[D, P_{a}\right]=-P_{a}, \quad[D, H]=-2 H, \quad\left[H, G_{a}\right]=P_{a} \quad\left[P_{a}, G_{b}\right]=\delta_{a b} N,} \\
& {\left[D, G_{a}\right]=G_{a}, \quad[D, K]=2 K, \quad\left[K, P_{a}\right]=-G_{a}, \quad[H, K]=D,} \\
& {\left[J_{a b}, P_{c}\right]=2 \delta_{c[a} P_{b]}, \quad\left[J_{a b}, G_{c}\right]=2 \delta_{c[a} G_{b]}, \quad\left[J_{a b}, J_{c d}\right]=4 \delta_{[a[c} J_{b] d]} .}
\end{aligned}
$$

The corresponding gauge fields and gauge parameters of each generator are given in table 5 . We split the transformation of a gauge field $A_{\mu}$ into a general coordinate transformation, with parameter $\xi^{\mu}$, and the other 'standard' gauge transformations according to

$$
\delta A_{\mu}=\delta_{\xi} A_{\mu}+\partial_{\mu} \epsilon+\left[A_{\mu}, \epsilon\right] .
$$

All gauge fields transform as covariant vectors under general coordinate transformations, so we usually only refer to the standard gauge transformation piece.

The time-like vielbein $\tau_{\mu}$, spatial vielbein $e_{\mu}{ }^{a}$, central charge gauge field $m_{\mu}$ and the temporal projection of the dilatation gauge field $b_{0}=\tau^{\mu} b_{\mu}$ are independent gauge fields whose transformation rules are given by

$$
\begin{aligned}
\delta \tau_{\mu} & =2 \Lambda_{D} \tau_{\mu}, \\
\delta e_{\mu}{ }^{a} & =\Lambda_{b}^{a} e_{\mu}{ }^{b}+\Lambda^{a} \tau_{\mu}+\Lambda_{D} e_{\mu}{ }^{a}, \\
\delta b_{0} & =\partial_{0} \Lambda_{D}+\Lambda_{K}-\Lambda^{a} b_{a}-2 \Lambda_{D} b_{0}, \\
\delta m_{\mu} & =\partial_{\mu} \sigma+\Lambda^{a} e_{\mu a} .
\end{aligned}
$$

We consider the case of twistless torsion, meaning that $\tau_{\mu}$ additionally satisfies

$$
e_{a}^{\mu} e_{b}^{\nu}\left(\partial_{\mu} \tau_{\nu}-\partial_{\nu} \tau_{\mu}\right)=0
$$

The remaining Schrödinger gauge fields are dependent and are solved for in terms of the independent ones by imposing curvature constraints whose formulae we do not give here. 
The dependent gauge fields $\omega_{\mu}^{a b}, \omega_{\mu}^{a}, b_{a}$ and $f_{a}$ are expressed in terms of $\tau_{\mu}, e_{\mu}^{a}, m_{\mu}$ and $b_{0}$ as follows:

$$
\begin{aligned}
\omega_{\mu}^{a b} & =\Omega_{\mu}^{a b}+2 e_{\mu}{ }^{[a} b^{b]}, \\
\omega_{\mu}{ }^{a} & =\Omega_{\mu}{ }^{a}+e_{\mu}{ }^{a} b_{0}, \\
b_{a} & =e_{a}{ }^{\mu} \tau^{\nu} \partial_{[\mu} \tau_{\nu]}, \\
f_{a} & =2 e_{a}{ }^{\mu} \tau^{\nu} \partial_{[\mu} b_{\nu]},
\end{aligned}
$$

where

$$
\begin{aligned}
\Omega_{\mu}{ }^{a b} & =-2 e^{\nu[a} \partial_{[\mu} e_{\nu]}^{b]}+e_{\mu c} e^{\nu a} e^{\rho b} \partial_{[\nu} e_{\rho]}^{c}-\tau_{\mu} e^{\nu a} e^{\rho b} \partial_{[\nu} m_{\rho]}, \\
\Omega_{\mu}{ }^{a} & =\tau^{\nu} \partial_{[\mu} e_{\nu]}^{a}+\tau^{\nu} e^{\rho a} e_{\mu b} \partial_{[\rho} e_{\nu]}^{b}+e^{\nu a} \partial_{[\mu} m_{\nu]}+\tau_{\mu} \tau^{\rho} e^{\nu a} \partial_{[\rho} m_{\nu]},
\end{aligned}
$$

are the rotation and boost gauge fields of the Bargmann algebra. The remaining dependent gauge field $f_{0}$ cannot be solved for using a fully gauge invariant constraint written purely in terms of Schrödinger gauge fields, see the discussion in [51]. However, using $M_{\mu}$, a vector transforming under boosts as in eq. (3.6), this problem can be circumvented and a gauge invariant constraint provides the solution:

$$
f_{0}=\frac{2}{d} \tau^{\mu} e^{\nu}{ }_{a}\left(D_{[\mu} \omega_{\nu]}^{a}+b_{[\mu} \omega_{\nu]}^{a}\right)+\frac{2}{d}\left(R_{0 a}^{a c}(J)+\frac{1}{2} M^{a} R_{a b}{ }^{b c}(J)\right) M_{c},
$$

where $D_{\mu}$ is the covariant derivative with respect to the Schrödinger spatial rotations and $R_{\mu \nu}^{a b}(J)$ is the Schrödinger spatial curvature defined in (A.10). Whenever in the text one of these dependent gauge fields occur, it is understood that they are given by the expressions above.

The transformation rules of the dependent gauge fields are given by

$$
\begin{aligned}
\delta \omega_{\mu}{ }^{a b} & =D_{\mu} \Lambda^{a b}, \\
\delta \omega_{\mu}{ }^{a} & =D_{\mu} \Lambda^{a}+\Lambda_{b}^{a} \omega_{\mu}{ }^{b}+\Lambda^{a} b_{\mu}-\Lambda_{D} \omega_{\mu}{ }^{a}+\Lambda_{K} e_{\mu}{ }^{a}, \\
\delta b_{a} & =\partial_{a} \Lambda_{D}-\Lambda_{D} b_{a}+\Lambda_{a}{ }^{b} b_{b}, \\
\delta f_{\mu} & =\partial_{\mu} \Lambda_{K}+2 \Lambda_{K} b_{\mu}-2 \Lambda_{D} f_{\mu} .
\end{aligned}
$$

The Schrödinger curvatures that we use explicitly in this work are the curvatures $R(J)$ associated to spatial rotations and $R(G)$ corresponding to boosts. These curvatures are given by

$$
\begin{aligned}
R_{\mu \nu}^{a b}(J) & =2 \partial_{[\mu} \omega_{\nu]}^{a b}-2 \omega_{[\mu}^{c[a} \omega_{\nu]}^{b]} c \\
R_{\mu \nu}{ }^{a}(G) & =2 \partial_{[\mu} \omega_{\nu]}^{a}+2{\omega_{[\mu}}^{b} \omega_{\nu]}^{a} b-2 \omega_{[\mu}^{a} b_{\nu]}-2 f_{[\mu} e_{\nu]}^{a} .
\end{aligned}
$$

We define the curvatures for the Bargmann rotation connection $\Omega_{\mu}^{a b}$ in (A.6) and for the Bargmann boost gauge field $\Omega_{\mu}{ }^{a}$ in (A.7) as follows:

$$
\begin{aligned}
& \mathcal{R}_{\mu \nu}{ }^{a b}(J)=2 \partial_{[\mu} \Omega_{\nu]}^{a b}-2 \Omega_{[\mu}{ }^{c[a} \Omega_{\nu]}{ }^{b]} c, \\
& \mathcal{R}_{\mu \nu}{ }^{a}(G)=2 \partial_{[\mu} \Omega_{\nu]}{ }^{a}+2 \Omega_{[\mu}{ }^{b} \Omega_{\nu]}{ }^{a} b .
\end{aligned}
$$


Formally, one can obtain the Bargmann quantities (A.6), (A.7) and (A.11) from the corresponding Schrödinger expressions (A.5a), (A.5b) and (A.10) by setting $b_{\mu}=f_{\mu}=0 .{ }^{21} \mathrm{We}$ will also use the Galilean covariant derivatives

$$
\begin{aligned}
\mathcal{D}_{0} b_{a} & =\tau^{\mu}\left(\partial_{\mu} b_{a}-\Omega_{\mu a}^{c} b_{c}\right), \\
\mathcal{D}_{a} b_{b} & =e_{a}^{\mu}\left(\partial_{\mu} b_{b}-\Omega_{\mu b}{ }^{c} b_{c}\right) \\
\mathcal{D}_{a} M_{b} & =e_{a}^{\mu}\left(\partial_{\mu} M_{b}-\Omega_{\mu b}{ }^{c} M_{c}-\Omega_{\mu b}\right) .
\end{aligned}
$$

Note that $\mathcal{D}_{a} b_{b}$ is a Galilean boost invariant by itself. This is unlike $\mathcal{D}_{0} b_{a}$ and $\mathcal{D}_{a} M_{b}$ which can however be used to construct a Galilean boost invariant in conjunction with additional terms. We find the Galilean boost invariant combinations:

$$
\begin{aligned}
K_{a} & =\mathcal{D}_{0} b_{a}+M^{b} \mathcal{D}_{b} b_{a}+b_{a} b_{b} M^{b}-M_{a} b \cdot b, \\
K_{a b} & =\mathcal{D}_{a} M_{b}+M_{a} b_{b}+M_{b} b_{a} .
\end{aligned}
$$

It follows from the definitions (A.4) and (A.6) that $\mathcal{D}_{[a} b_{b]}=0$. Furthermore, from the same equations (A.4) and (A.6) it can be seen that, as a consequence of having twistless torsion, the term $\mathcal{D} \cdot b \equiv \delta^{a b} \mathcal{D}_{a} b_{b}$ is a total derivative only up to an additional torsion contribution, namely:

$$
\mathcal{D} \cdot b+2 b \cdot b=e^{-1} \partial_{\mu}\left(e e_{a}^{\mu} b^{a}\right) .
$$

Using the definitions (A.6) and (A.7) one may also verify that the anti-symmetric part of $\mathcal{D}_{a} M_{b}$ is vanishing

$$
\mathcal{D}_{[a} M_{b]}=e_{a}^{\mu} e_{b}^{\nu}\left(\partial_{[\mu} M_{\nu]}-\partial_{[\mu} m_{\nu]}\right)=0 .
$$

To show the last step, one uses the relation between $M_{\mu}$ and $m_{\mu}$ given in (3.9).

\section{B Scalar Schrödinger field theories}

In this appendix we classify all possible independent complex scalar field theories invariant under rigid $z=2$ Schrödinger transformations up to second order in time-derivatives and fourth order in spatial-derivatives. Explicitly, the complex scalar field $\Psi$ transforms according to eq. (3.14) with the parameters given by eqs. (3.13) as follows:

$$
\begin{aligned}
\delta \Psi= & {\left[\xi^{0} \partial_{0}+\xi^{a} \partial_{a}+w \Lambda_{D}+i \mathrm{M} \sigma\right] \Psi } \\
= & \left(a^{0}-2 \lambda_{D} t+\lambda_{K} t^{2}\right) \partial_{0} \Psi+\left(a^{c}-\lambda^{c b} x_{b}-\lambda^{c} t-\lambda_{D} x^{c}+\lambda_{K} t x^{c}\right) \partial_{c} \Psi \\
& +w\left(\lambda_{D}-\lambda_{K} t\right) \Psi+i \mathrm{M}\left(\sigma_{0}-\lambda^{a} x_{a}+\frac{1}{2} \lambda_{K} x^{2}\right) \Psi .
\end{aligned}
$$

Here all parameters are constants, $w$ is the dilatation weight and $\mathrm{M}$ is the weight under central charge transformations.

\footnotetext{
${ }^{21}$ We notice that in describing the Newton-Cartan geometry the vielbeine $\left(\tau_{\mu}\right)^{\mathrm{G}}$ and $\left(e_{\mu}^{a}\right)^{\mathrm{G}}$ are in general not the same as the Schrödinger ones. They are only the same after gauge fixing, see eq. (3.9).
} 
The independent scalar SFT's we will obtain are only defined up to boundary terms. However, because ultimately we are interested in coupling these scalar field theories to construct local invariants, we will fix this ambiguity by performing our classification directly at the level of the Lagrangian. We recall that, strictly speaking, the Lagrangians are never invariant under the transformation (B.1). Hence, we will classify the Lagrangians by imposing that they transform according to the following total derivative:

$$
\delta \mathcal{L}=\partial_{0}\left(\xi^{0} \mathcal{L}\right)+\partial_{a}\left(\xi^{a} \mathcal{L}\right)
$$

With slight abuse of terminology we will refer to a Lagrangian satisfying (B.2) as an invariant Lagrangian. This condition guaranties that the action will be invariant under the full Schödinger transformations (B.1) and that the Lagrangian admits a coupling to Schrödinger gravity, see also the discussion just above and below equation (2.22). In general, the boundary terms generated by performing partial integrations are not invariant by themselves. Therefore, two Lagrangians that are related by such a boundary term will not both be invariant upon throwing away the boundary term and this fixes one preferred Lagrangian over the other. As we will see next, in one specific case the boundary term is guaranteed to be an invariant. In this case, the analysis can be performed directly at the level of the action.

The way we organize the classification goes as follow. We start in subsection B.1 with the classification of the potential terms, i.e. terms without time-derivatives, and in subsection B.2 we consider the more involved case of Schrödinger invariant actions with time-derivatives. Depending on what is most convenient we will use a formulation where the derivatives act on the complex scalar $\Psi$ and its conjugate $\Psi^{\star}$ or on the two real scalars $\varphi$ and $\chi$. We recall that

$$
\Psi=\varphi e^{i \chi} .
$$

We will first fix the most general terms that can be written down by requiring invariance under dilatation and central charge symmetry. In a second step we will add the required compensating terms to form invariants under Galilean boost and special conformal symmetries.

In principle the analysis can be done for arbitrary spatial dimensions $d$ and arbitrary dilatation weight $w$. However, it can be seen that the SFT's we obtain are significantly simplified by fixing the dilatation weight to

$$
w=-\frac{d+2-2 n_{t}-n_{s}}{2},
$$

where $n_{t}$ is the number of time derivatives and $n_{s}$ the number of spatial derivatives. In order to improve the presentation of our results we will therefore fix the weight according to (B.4) from the start. Note that by construction we are assuming $w \neq 0$. Strictly speaking, the condition (B.4) is therefore a condition on the spatial dimension. However, nothing depends crucially on the choice (B.4) and it is straightforward to include the case $d=2 n_{t}+n_{s}-2$ by fixing $w$ differently. 


\section{B.1 Potential terms}

We start our classification by the potential terms. In this case we write the complex scalar $\Psi$ in terms of its norm $\varphi$ and angle $\chi$. In a first step, the construction of invariant potential terms is simplified by letting the spatial derivatives act only on the norm $\varphi$. This case is very special: provided all indices are contracted we are guaranteed to produce an invariant. For that reason also the possible boundary terms created by partial integrations are invariants by themselves. This means that in this case it is sufficient to perform the classification at the level of the action. This first part of our analysis is therefore equivalent to the classification of all inequivalent ways to contract the spatial indices of the derivatives acting on $\varphi$ up to boundary terms. In a second step we look at possible invariants where a spatial derivative is also allowed to act on $\chi$. We will find that there are no such term with two spatial derivatives and only one at the next order with four derivatives.

At zeroth order, with $n_{t}=n_{s}=0$, we fix the dilatation weight to $-\frac{d+2}{2}$ according to (B.4). It follows that the only Schrödinger invariant action takes the form

$$
\mathrm{SFT}_{0}: S^{(0)}=\int d t d^{d} x \Lambda_{0} \varphi^{2}
$$

where $\Lambda_{0}$ is an arbitrary function.

At second order $\left(n_{t}=0, n_{s}=2\right)$, with spatial derivatives acting only on $\varphi$, the dilatation symmetry implies that we can only write down two terms in the Lagrangian: $\partial^{a} \varphi \partial_{a} \varphi$ and $\varphi \partial^{a} \partial_{a} \varphi$. All other symmetries are then automatically satisfied. Moreover, these two terms are related by an invariant boundary term, hence there is no difference between using one or the other. ${ }^{22}$ Therefore, we find that the unique Schrödinger field theory at this order is

$$
\mathrm{SFT}_{1}: \quad S^{(1)}=\int d t d^{d} x \partial^{a} \varphi \partial_{a} \varphi
$$

We recall that the weight of the scalar field $\varphi$ is fixed by (B.4).

Performing the same analysis at fourth order in the spatial derivatives $\left(n_{t}=0, n_{s}=4\right)$, we find three independent invariant SFT's given by

$$
\begin{aligned}
& \mathrm{SFT}_{2}: \quad S^{(2)}=\int d t d^{d} x \varphi^{-2}\left(\partial^{a} \varphi \partial_{a} \varphi\right)^{2}, \\
& \mathrm{SFT}_{3}: \quad S^{(3)}=\int d t d^{d} x \varphi^{-1}\left(\partial^{a} \varphi \partial_{a} \varphi\right)\left(\partial^{b} \partial_{b} \varphi\right), \\
& \mathrm{SFT}_{4}: \quad S^{(4)}=\int d t d^{d} x\left(\partial^{a} \partial_{a} \varphi\right)^{2} .
\end{aligned}
$$

These correspond to the only three possible actions that can be built with four spatial derivatives acting on $\varphi$ and that cannot be related by partial integrations.

Let us now analyze whether there are also invariant potential terms involving the real scalar field $\chi$. First of all, note that if we write a term where $\chi$ appears without any derivatives the Lagrangian cannot be made invariant under central charge symmetry.

\footnotetext{
${ }^{22}$ In section 5 we confirm that both terms do indeed lead to the same action after coupling to Schrödinger gravity.
} 
Furthermore, the scalar field $\chi$ is odd under time reversal. Because we consider only spacial derivatives we need $\chi$ to appear an even number of times in order to produce time reversal invariant field theories.

With two spatial derivatives the only term that we can write down with correct scaling behavior is: $\varphi^{2} \partial^{a} \chi \partial_{a} \chi$. However, this term by itself is not an invariant and there is nothing else that can be written down that could be added to make it invariant. We conclude that (B.6) is the only invariant at second order in spatial derivatives. ${ }^{23}$

At fourth order the situation is more interesting. Imposing central charge, dilatation and time reversal symmetry, we are in principle allowed to consider the following list of terms:

$$
\begin{aligned}
& \varphi \partial^{a} \partial_{a} \varphi \partial_{b} \chi \partial^{b} \chi, \varphi \partial_{a} \partial_{b} \varphi \partial^{a} \chi \partial^{b} \chi, \partial^{a} \varphi \partial_{a} \varphi \partial_{b} \chi \partial^{b} \chi, \partial_{a} \varphi \partial_{b} \varphi \partial^{a} \chi \partial^{b} \chi, \\
& \varphi \partial_{a} \varphi \partial^{a} \chi \partial^{b} \partial_{b} \chi, \varphi \partial_{a} \varphi \partial^{a} \partial^{b} \chi \partial_{b} \chi, \\
& \varphi^{2} \partial_{a} \chi \partial^{a} \chi \partial_{b} \chi \partial^{b} \chi, \varphi^{2} \partial^{a} \partial_{a} \chi \partial_{b} \partial^{b} \chi, \varphi^{2} \partial^{a} \partial^{b} \chi \partial_{a} \partial_{b} \chi, \varphi^{2} \partial^{a} \partial_{a} \partial^{b} \chi \partial_{b} \chi,
\end{aligned}
$$

respectively with two, three and four derivatives acting on $\chi$. Taking the most general linear combination of the terms in (B.8) we find a unique Schrödinger invariant given by

$$
\mathrm{SFT}_{6^{\prime}}: \quad S^{\left(6^{\prime}\right)}=\int d t d^{d} x \varphi^{2}\left(\left(\partial^{a} \partial_{a} \chi\right)^{2}-d \partial_{a} \partial_{b} \chi \partial^{a} \partial^{b} \chi\right) .
$$

Interestingly, although this is a potential term, after coupling to Schrödinger gravity and gauge fixing this invariant does generate a linear combination of the HL kinetic terms. However, we do not consider it in the main text where we prefer to work with another set of independent invariants generated by the complex field $\Psi$ (see section B.2). This is the reason why we added a prime on the label. This will become clearer in the next section when we give the explicit relation between the invariant (B.9) and the ones we will use effectively. This concludes our discussion of the potential terms.

\section{B.2 Kinetic terms}

We now turn to the construction of scalar SFT's that contain time derivatives. In this case we will mostly work with the derivatives acting on the fields $\Psi$ and $\Psi^{\star}$ in order to make contact with the Schrödinger action in its most well-known form.

We start by looking at possible invariant theories containing a single time derivative ( $\left.n_{t}=1, n_{s}=0\right)$ where, we can without loss of generality fix the dilatation weight according to eq. (B.4) to $w=-\frac{d}{2}$. Imposing invariance under central charge and dilatation symmetry there are only two terms that we can write down with a single time derivative: $\Psi^{\star} \partial_{0} \Psi$ and its complex conjugate $\Psi \partial_{0} \Psi^{\star}$. Let us consider $\Psi^{\star} \partial_{0} \Psi$, by computing the variation (B.1) on this term we obtain

$$
\delta\left(\Psi^{\star} \partial_{0} \Psi\right) \cong-\Psi^{\star}\left(\lambda^{a} \partial_{a}+\lambda_{K}\left(w-x^{a} \partial_{a}\right)\right) \Psi
$$

where by $\cong$ we mean equality up to the total derivative of equation (B.2). The terms on the right hand side of (B.10) can be exactly compensated for by the addition on the

\footnotetext{
${ }^{23}$ This would also be the case even without imposing time reversal invariance.
} 
left hand side of extra terms containing only spatial derivatives. The resulting invariant combination is the Lagrangian describing the Schrödinger action,

$$
\mathrm{SFT}_{5}: \quad S^{(5)}=\int d t d^{d} x \Psi^{\star}\left(i \partial_{0}-\frac{1}{2 \mathrm{M}} \partial^{a} \partial_{a}\right) \Psi
$$

This is the action that we will couple to Schrödinger gravity in section 5.1.2.

Although the Lagrangian of eq. (B.11) has an imaginary part, the Schrödinger action itself is real. Performing a similar analysis starting with $\Psi \partial_{0} \Psi^{\star}$ we would just find the complex conjugate of the Lagrangian of equation (B.11), leading to nothing new. Therefore, we conclude that, up to invariant potential terms, the Schrödinger action (B.11) is the unique invariant at first order in time derivative.

We next consider SFT's at second order in time derivatives and following (B.4) we fix the dilatation weight to $w=-\frac{d-2}{2}$. Here the possibilities increase. Imposing dilatation and central charge invariance leads to the following five possible kinetic terms

$$
\Psi^{\star} \partial_{0}^{2} \Psi, \Psi \partial_{0}^{2} \Psi^{\star}, \partial_{0} \Psi \partial_{0} \Psi^{\star}, \Psi^{\star} \Psi^{-1}\left(\partial_{0} \Psi\right)^{2}, \Psi \Psi^{\star-1}\left(\partial_{0} \Psi^{\star}\right)^{2}
$$

We now perform a similar analysis as in the first order case. Up to complex conjugation there are three cases to consider.

(1) For the term $\Psi^{\star} \partial_{0}^{2} \Psi$, we find that its variation

$$
\delta\left(\Psi^{\star} \partial_{0}^{2} \Psi\right) \cong-2 \Psi^{\star}\left(\lambda^{a} \partial_{a} \partial_{0}+\lambda_{K}\left((w-1) \partial_{0}-x^{a} \partial_{a} \partial_{0}\right)\right) \Psi
$$

can be exactly compensated for by the addition of extra terms. The corresponding invariant Lagrangian is leading to the action

$$
\mathrm{SFT}_{6}: \quad S^{(6)}=\int d t d^{d} x \Psi^{\star}\left(i \partial_{0}-\frac{1}{2 \mathrm{M}} \partial^{a} \partial_{a}\right)^{2} \Psi,
$$

which we recognize as the square of (B.11). For the same reasons as in the first order case, the complex conjugate $\Psi \partial_{0}^{2} \Psi^{\star}$ cannot give anything new.

(2) The variation of the next possible term at this order, namely $\partial_{0} \Psi \partial_{0} \Psi^{\star}$, is

$$
\delta\left(\partial_{0} \Psi \partial_{0} \Psi^{\star}\right) \cong\left(\lambda_{K} x^{a}-\lambda^{a}\right)\left(\partial_{a} \Psi^{\star} \partial_{0} \Psi+\partial_{a} \Psi \partial_{0} \Psi^{\star}\right)-w \lambda_{K}\left(\Psi^{\star} \partial_{0} \Psi+\Psi \partial_{0} \Psi^{\star}\right) .
$$

Again, the variation above can be compensated for leading to a unique invariant Lagrangian:

$$
\mathrm{SFT}_{7}: \quad S^{(7)}=\int d t d^{d} x\left|\left(i \partial_{0}-\frac{1}{2 \mathrm{M}} \partial^{a} \partial_{a}\right) \Psi+\frac{1}{\mathrm{M} d}\left(\partial^{a} \partial_{a} \Psi-\frac{1}{\Psi}\left(\partial_{a} \Psi\right)^{2}\right)\right|^{2},
$$

where $|\cdot|$ is the norm. 
(3) Proceeding in a similar way with the kinetic term $\Psi^{\star} \Psi^{-1}\left(\partial_{0} \Psi\right)^{2}$ it turns out that its variation:

$$
\delta\left(\Psi^{\star} \Psi^{-1}\left(\partial_{0} \Psi\right)^{2}\right) \cong-2 \Psi^{\star} \partial_{0} \Psi\left(w \lambda_{K}+\Psi^{-1}\left(\lambda^{a}-x^{a} \lambda_{K}\right) \partial_{a} \Psi\right),
$$

cannot directly be compensated for. In this case, it is necessary to combine this term with its complex conjugate. In order to simplify the final interpretation of this action we will find it convenient to actually consider the variation of the following combination of terms

$$
-\Psi^{\star} \frac{\left(\partial_{0} \Psi\right)^{2}}{\Psi}-\Psi \frac{\left(\partial_{0} \Psi^{\star}\right)^{2}}{\Psi^{\star}}+2 \partial_{0} \Psi \partial_{0} \Psi^{\star}=-\varphi^{-2}\left(\Psi^{\star} \partial_{0} \Psi-\Psi \partial_{0} \Psi^{\star}\right)^{2},
$$

where an additional $\partial_{0} \Psi \partial_{0} \Psi^{\star}$ term has been added to complete the square. After compensating the variation of the combination in (B.18) we find the following invariant action

$$
\mathrm{SFT}_{8}: \quad S^{(8)}=\int d t d x^{a} \frac{1}{\Psi^{\star} \Psi}\left(i \Psi^{\star} \partial_{0} \Psi-i \Psi \partial_{0} \Psi^{\star}+\frac{1}{\mathrm{M}} \partial_{a} \Psi \partial_{a} \Psi^{\star}\right)^{2} .
$$

We can recognize this action as the square of the partially integrated Schrödinger action, see also the discussion above (5.18) in the main text. This exhausts all the possibilities and concludes our discussion of the kinetic terms with two time derivatives.

At last, we look at possible invariant with one time and two spatial derivatives in terms of $\varphi$ and $\chi$. Imposing central charge, dilatation and time-reversal symmetry the only terms that can be written down are

$$
\begin{aligned}
& \varphi^{2} \partial_{0} \partial^{2} \chi, \varphi^{2} \partial_{0} \chi \partial^{a} \chi \partial_{a} \chi, \varphi \partial_{0} \varphi \partial^{2} \chi, \varphi \partial^{a} \varphi \partial_{0} \partial_{a} \chi, \\
& \varphi \partial_{0} \partial^{a} \varphi \partial_{a} \chi, \varphi \partial^{2} \varphi \partial_{0} \chi, \partial_{0} \varphi \partial^{a} \varphi \partial_{a} \chi, \partial^{a} \varphi \partial_{a} \varphi \partial_{0} \chi
\end{aligned}
$$

where we recall that the dilatation weight is fixed to $w=-\frac{d-2}{2}$. From the terms in (B.20) it is possible to build three invariant Lagrangians:

$$
\begin{aligned}
& I_{1}=\partial^{a} \varphi \partial_{a} \varphi \partial_{0} \chi-\frac{1}{2 \mathrm{M}}\left(\partial^{a} \varphi \partial_{a} \varphi \partial^{b} \chi \partial_{b} \chi\right), \\
& I_{2}=\varphi \partial_{a} \partial^{a} \varphi \partial_{0} \chi-\frac{1}{2 \mathrm{M}}\left(\varphi \partial_{a} \partial^{a} \varphi \partial^{b} \chi \partial_{b} \chi\right), \\
& I_{3}=\varphi \partial^{a} \varphi \partial_{0} \partial_{a} \chi-\frac{1}{\mathrm{M}}\left(\varphi \partial^{a} \varphi \partial^{b} \chi \partial_{a} \partial_{b} \chi\right) .
\end{aligned}
$$

However, it can be seen that these Lagrangians lead to only two linearly independent actions as $I_{3}$ is nothing but a combination of $I_{1}$ and $I_{2}$ up to a partial integration using $\partial_{a}$. The invariant Lagrangians (B.21a) and (B.21b) can be rewritten as the following invariant actions

$$
\begin{aligned}
\mathrm{SFT}_{9}: & S^{(9)}=\int d t d^{d} x \frac{1}{\varphi^{2}} \partial^{a} \varphi \partial_{a} \varphi\left(i \Psi^{\star} \partial_{0} \Psi-i \Psi \partial_{0} \Psi^{\star}+\frac{1}{\mathrm{M}} \partial^{a} \Psi^{\star} \partial_{a} \Psi\right), \\
\mathrm{SFT}_{10}: & S^{(10)}=\int d t d^{d} x \frac{1}{\varphi} \partial^{2} \varphi\left(i \Psi^{\star} \partial_{0} \Psi-i \Psi \partial_{0} \Psi^{\star}+\frac{1}{\mathrm{M}} \partial^{a} \Psi^{\star} \partial_{a} \Psi\right),
\end{aligned}
$$


where for convenience we have ignored an overall factor of $-\frac{1}{2}$ and added the invariant potential terms $\frac{1}{2} S^{(2)}$ and $\frac{1}{2} S^{(3)}$ to (B.21a) and (B.21b) respectively. The actions (B.22a) and (B.22b) are the ones we will couple to Schrödinger gravity in the main text.

Up to the order we are working $\left(n_{t} \leq 2\right.$ and $\left.n_{s} \leq 4\right)$, it is not possible to form any additional SFT that is linearly independent from the ones we obtained above in the set of $\mathrm{SFT}_{1-10}$. Finally, we come back to the invariant $\mathrm{SFT}_{6^{\prime}}$ that we found in eq. (B.9) and show that it is not an independent invariant. This follows from the relation:

$$
S^{\left(6^{\prime}\right)}=-d^{2} \mathrm{M}^{2}\left(S^{(6)}-S^{(7)}\right)-2 d \mathrm{M} S^{(10)}+(d-1) S^{(4)}+(d+2) S^{(3)}-S^{(2)} .
$$

This is the reason why we added a prime on its label. We do not couple the $\mathrm{SFT}_{6^{\prime}}$ in the main text where we choose to work with the set of $\mathrm{SFT}_{1-10}$ constructed from $\Psi$ and $\varphi$.

Open Access. This article is distributed under the terms of the Creative Commons Attribution License (CC-BY 4.0), which permits any use, distribution and reproduction in any medium, provided the original author(s) and source are credited.

\section{References}

[1] M. Kaku, P.K. Townsend and P. van Nieuwenhuizen, Properties of Conformal Supergravity, Phys. Rev. D 17 (1978) 3179 [INSPIRE].

[2] M. Kaku and P.K. Townsend, Poincaré Supergravity as Broken Superconformal Gravity, Phys. Lett. B 76 (1978) 54 [InSPIRE].

[3] S. Ferrara, M. Kaku, P.K. Townsend and P. van Nieuwenhuizen, Gauging the Graded Conformal Group with Unitary Internal Symmetries, Nucl. Phys. B 129 (1977) 125 [INSPIRE].

[4] M. Kaku, P.K. Townsend and P. van Nieuwenhuizen, Gauge Theory of the Conformal and Superconformal Group, Phys. Lett. B 69 (1977) 304 [InSPIRE].

[5] D.Z. Freedman and A. Van Proeyen, Supergravity, Cambridge University Press, Cambridge U.K. (2012).

[6] E. Cartan, Sur les variétés à connexion affine et la théorie de la relativité généralisée (première partie), Annales Sci. Ecole Norm. Sup. 40 (1923) 325.

[7] E. Cartan, Sur les variétés à connexion affine et la théorie de la relativité généralisée. (première partie) (Suite), Annales Sci. Ecole Norm. Sup. 41 (1924) 1.

[8] P. Hořava, Quantum Gravity at a Lifshitz Point, Phys. Rev. D 79 (2009) 084008 [arXiv:0901.3775] [INSPIRE].

[9] P. Hořava, Membranes at Quantum Criticality, JHEP 03 (2009) 020 [arXiv:0812.4287] [INSPIRE].

[10] G. Dautcourt, On the newtonian limit of general relativity, Acta Phys. Polon. B21 (1990) 755 .

[11] S. Mukohyama, Hořava-Lifshitz Cosmology: A Review, Class. Quant. Grav. 27 (2010) 223101 [arXiv: 1007.5199] [INSPIRE].

[12] T.P. Sotiriou, Hořava-Lifshitz gravity: a status report, J. Phys. Conf. Ser. 283 (2011) 012034 [arXiv: 1010.3218] [INSPIRE]. 
[13] J. Hartong and N.A. Obers, Hořava-Lifshitz gravity from dynamical Newton-Cartan geometry, JHEP 07 (2015) 155 [arXiv: 1504.07461] [INSPIRE].

[14] A. Trautman, Sur la theorie newtonienne de la gravitation, Compt. Rend. Acad. Sci. 257 (1963) 617.

[15] H.P. Kuenzle, Galilei and Lorentz structures on space-time - comparison of the corresponding geometry and physics, Annales Poincaré Phys. Theor. 17 (1972) 337 [InSPIRE].

[16] C. Duval, G. Burdet, H.P. Kunzle and M. Perrin, Bargmann Structures and Newton-cartan Theory, Phys. Rev. D 31 (1985) 1841 [INSPIRE].

[17] C. Leiva and M.S. Plyushchay, Conformal symmetry of relativistic and nonrelativistic systems and AdS/CFT correspondence, Annals Phys. 307 (2003) 372 [hep-th/0301244] [INSPIRE].

[18] K. Balasubramanian and J. McGreevy, Gravity duals for non-relativistic CFTs, Phys. Rev. Lett. 101 (2008) 061601 [arXiv: 0804.4053] [INSPIRE].

[19] D.T. Son, Toward an AdS/cold atoms correspondence: A Geometric realization of the Schrödinger symmetry, Phys. Rev. D 78 (2008) 046003 [arXiv: 0804.3972] [INSPIRE].

[20] C.P. Herzog, M. Rangamani and S.F. Ross, Heating up Galilean holography, JHEP 11 (2008) 080 [arXiv: 0807.1099] [INSPIRE].

[21] C. Duval, M. Hassaine and P.A. Horvathy, The Geometry of Schrödinger symmetry in gravity background/non-relativistic CFT, Annals Phys. 324 (2009) 1158 [arXiv:0809.3128] [INSPIRE].

[22] S. Kachru, X. Liu and M. Mulligan, Gravity duals of Lifshitz-like fixed points, Phys. Rev. D 78 (2008) 106005 [arXiv:0808.1725] [InSPIRE].

[23] A. Bagchi and R. Gopakumar, Galilean Conformal Algebras and AdS/CFT, JHEP 07 (2009) 037 [arXiv: 0902.1385] [INSPIRE].

[24] M. Taylor, Non-relativistic holography, arXiv:0812.0530 [INSPIRE].

[25] S. Janiszewski and A. Karch, Non-relativistic holography from Hořava gravity, JHEP 02 (2013) 123 [arXiv:1211.0005] [inSPIRE].

[26] T. Griffin, P. Hořava and C.M. Melby-Thompson, Lifshitz Gravity for Lifshitz Holography, Phys. Rev. Lett. 110 (2013) 081602 [arXiv:1211.4872] [InSPIRE].

[27] D.M. Hofman and B. Rollier, Warped Conformal Field Theory as Lower Spin Gravity, Nucl. Phys. B 897 (2015) 1 [arXiv:1411.0672] [INSPIRE].

[28] A. Castro, D.M. Hofman and N. Iqbal, Entanglement Entropy in Warped Conformal Field Theories, JHEP 02 (2016) 033 [arXiv:1511.00707] [INSPIRE].

[29] K. Jensen, On the coupling of Galilean-invariant field theories to curved spacetime, arXiv: 1408.6855 [INSPIRE].

[30] K. Jensen, Aspects of hot Galilean field theory, JHEP 04 (2015) 123 [arXiv:1411.7024] [INSPIRE].

[31] J. Hartong, E. Kiritsis and N.A. Obers, Schrödinger Invariance from Lifshitz Isometries in Holography and Field Theory, Phys. Rev. D 92 (2015) 066003 [arXiv:1409.1522] [INSPIRE].

[32] J. Hartong, E. Kiritsis and N.A. Obers, Field Theory on Newton-Cartan Backgrounds and Symmetries of the Lifshitz Vacuum, JHEP 08 (2015) 006 [arXiv:1502.00228] [InSPIRE]. 
[33] M. Geracie, K. Prabhu and M.M. Roberts, Curved non-relativistic spacetimes, Newtonian gravitation and massive matter, J. Math. Phys. 56 (2015) 103505 [arXiv:1503.02682] [INSPIRE].

[34] M. Geracie, K. Prabhu and M.M. Roberts, Fields and fluids on curved non-relativistic spacetimes, JHEP 08 (2015) 042 [arXiv:1503.02680] [INSPIRE].

[35] D.T. Son, Newton-Cartan Geometry and the Quantum Hall Effect, arXiv:1306.0638 [INSPIRE].

[36] A. Gromov and A.G. Abanov, Thermal Hall Effect and Geometry with Torsion, Phys. Rev. Lett. 114 (2015) 016802 [arXiv: 1407.2908] [INSPIRE].

[37] M. Geracie, D.T. Son, C. Wu and S.-F. Wu, Spacetime Symmetries of the Quantum Hall Effect, Phys. Rev. D 91 (2015) 045030 [arXiv:1407.1252] [InSPIRE].

[38] S. Moroz and C. Hoyos, Effective theory of two-dimensional chiral superfluids: gauge duality and Newton-Cartan formulation, Phys. Rev. B 91 (2015) 064508 [arXiv:1408.5911] [INSPIRE].

[39] A.J. Bray, Theory of phase-ordering kinetics, Adv. Phys. 43 (1994) 357 [InSPIRE].

[40] M. Henkel, M. Pleimling, C. Godreche and J.-M. Luck, Aging and conformal invariance, Phys. Rev. Lett. 87 (2001) 265701 [hep-th/0107122] [INSPIRE].

[41] M. Henkel, Phenomenology of local scale invariance: From conformal invariance to dynamical scaling, Nucl. Phys. B 641 (2002) 405 [hep-th/0205256] [InSPIRE].

[42] M. Henkel and F. Baumann, Autocorrelation functions in phase-ordering kinetics from local scale-invariance, J. Stat. Mech. 0707 (2007) P07015 [cond-mat/0703226] [INSPIRE].

[43] M. Henkel and M. Pleimling, Non-Equilibrium Phase Transitions. Volume 2: Ageing and Dynamical Scaling Far from Equilibrium, Springer, Dordrecht Netherlands (2010).

[44] D. Minic and M. Pleimling, Non-relativistic AdS/CFT and Aging/Gravity Duality, Phys. Rev. E 78 (2008) 061108 [arXiv:0807.3665] [INSPIRE].

[45] J.I. Jottar, R.G. Leigh, D. Minic and L.A. Pando Zayas, Aging and Holography, JHEP 11 (2010) 034 [arXiv: 1004.3752] [INSPIRE].

[46] D. Minic, D. Vaman and C. Wu, On the 3-point functions of Aging Dynamics and the AdS/CFT Correspondence, Phys. Rev. Lett. 109 (2012) 131601 [arXiv:1207.0243] [INSPIRE].

[47] M.H. Christensen, J. Hartong, N.A. Obers and B. Rollier, Boundary Stress-Energy Tensor and Newton-Cartan Geometry in Lifshitz Holography, JHEP 01 (2014) 057 [arXiv:1311.6471] [INSPIRE].

[48] M.H. Christensen, J. Hartong, N.A. Obers and B. Rollier, Torsional Newton-Cartan Geometry and Lifshitz Holography, Phys. Rev. D 89 (2014) 061901 [arXiv:1311.4794] [INSPIRE].

[49] M. Taylor, Lifshitz holography, Class. Quant. Grav. 33 (2016) 033001 [arXiv:1512.03554] [INSPIRE].

[50] R. Andringa, E. Bergshoeff, S. Panda and M. de Roo, Newtonian Gravity and the Bargmann Algebra, Class. Quant. Grav. 28 (2011) 105011 [arXiv:1011.1145] [InSPIRE].

[51] E.A. Bergshoeff, J. Hartong and J. Rosseel, Torsional Newton-Cartan geometry and the Schrödinger algebra, Class. Quant. Grav. 32 (2015) 135017 [arXiv: 1409.5555] [INSPIRE]. 
[52] C.R. Hagen, Scale and conformal transformations in galilean-covariant field theory, Phys. Rev. D 5 (1972) 377 [INSPIRE].

[53] U. Niederer, The maximal kinematical invariance group of the free Schrödinger equation, Helv. Phys. Acta 45 (1972) 802 [inSPIRE].

[54] A.O. Barut, Conformal Group $\rightarrow$ Schrödinger Group $\rightarrow$ Dynamical Group - The Maximal Kinematical Group of the Massive Schrödinger Particle, Helv. Phys. Acta 46 (1973) 496.

[55] P. Havas and J. Plebánski, Conformal extensions of the Galilei group and their relation to the Schrödinger group., J. Math. Phys 19 (1978) 482.

[56] R. Jackiw and S.-Y. Pi, Classical and quantal nonrelativistic Chern-Simons theory, Phys. Rev. D 42 (1990) 3500 [Erratum ibid. D 48 (1993) 3929] [INSPIRE].

[57] M. Henkel, Schrödinger invariance in strongly anisotropic critical systems, J. Statist. Phys. 75 (1994) 1023 [hep-th/9310081] [INSPIRE].

[58] Y. Nishida and D.T. Son, Nonrelativistic conformal field theories, Phys. Rev. D 76 (2007) 086004 [arXiv: 0706.3746] [INSPIRE].

[59] R. Banerjee, A. Mitra and P. Mukherjee, Localization of the Galilean symmetry and dynamical realization of Newton-Cartan geometry, Class. Quant. Grav. 32 (2015) 045010 [arXiv: 1407 . 3617] [INSPIRE].

[60] R. Banerjee and P. Mukherjee, New approach to nonrelativistic diffeomorphism invariance and its applications, arXiv: 1509.05622 [INSPIRE].

[61] R. De Pietri, L. Lusanna and M. Pauri, Standard and generalized Newtonian gravities as 'gauge' theories of the extended Galilei group. I. The standard theory, Class. Quant. Grav. 12 (1995) 219 [gr-qc/9405046] [INSPIRE].

[62] C. Duval and H.P. Kunzle, Minimal Gravitational Coupling in the Newtonian Theory and the Covariant Schrödinger Equation, Gen. Rel. Grav. 16 (1984) 333 [INSPIRE].

[63] J. Ehlers, Über den Newtonschen Grenzwert der Einsteinschen Gravitationstheorie, in Grundlagenprobleme der modernen Physik, J. Nitsch, J. Pfarr and E.W. Stachow eds., Bibliographisches Institut, Mannheim Germany (1981), pp. 65-84.

[64] E. Bergshoeff, J. Rosseel and T. Zojer, Newton-Cartan (super)gravity as a non-relativistic limit, Class. Quant. Grav. 32 (2015) 205003 [arXiv: 1505.02095] [INSPIRE].

[65] D. Blas, O. Pujolàs and S. Sibiryakov, Models of non-relativistic quantum gravity: The Good, the bad and the healthy, JHEP 04 (2011) 018 [arXiv:1007.3503] [INSPIRE].

[66] D. Blas, O. Pujolàs and S. Sibiryakov, Consistent Extension of Hor̆ava Gravity, Phys. Rev. Lett. 104 (2010) 181302 [arXiv:0909.3525] [INSPIRE].

[67] T.P. Sotiriou, M. Visser and S. Weinfurtner, Phenomenologically viable Lorentz-violating quantum gravity, Phys. Rev. Lett. 102 (2009) 251601 [arXiv:0904.4464] [INSPIRE].

[68] E. Bergshoeff, J. Rosseel and T. Zojer, Newton-Cartan supergravity with torsion and Schrödinger supergravity, JHEP 11 (2015) 180 [arXiv: 1509.04527] [INSPIRE].

[69] R. Andringa, E.A. Bergshoeff, J. Rosseel and E. Sezgin, 3D Newton-Cartan supergravity, Class. Quant. Grav. 30 (2013) 205005 [arXiv: 1305.6737] [INSPIRE].

[70] K. Peeters, A Field-theory motivated approach to symbolic computer algebra, Comput. Phys. Commun. 176 (2007) 550 [cs/0608005] [INSPIRE]. 